\title{
Etude comparée des quantités ingérées et du comportement alimentaire et mérycique d'ovins et de bovins recevant des ensilages d'herbe réalisés selon différentes techniques
}

\author{
J.P. DULPHY, Brigitte MICHALET-DOREAU et C. DEMARQUILLY \\ I.N.R.A., Laboratoire des Aliments, C.R.Z.V. de Theix \\ F 63122 Ceyrat
}

avec la collaboration technique de H. Bousquet, L. L'Hotelier, Marie JAiller, Jacqueline Jamot et Madeleine DudiLIEU

\begin{abstract}
Résumé
Vingt ensilages d'herbe, préparés selon différentes techniques à partir de 10 fourrages verts, ont été distribués à des moutons et à des génisses durant la période hivernale. Les mesures effectuées concernent les quantités ingérées, le comportement alimentaire et la rumination.

Les génisses ont ingéré certains ensilages en quantité équivalente aux fourrages verts, ce qui n'a pas été le cas pour les moutons. Elles sont en outre moins sensibles que ces derniers à l'allongement des brins d'ensilage.

Les paramètres qui expliquent le mieux les variations de quantités ingérées d'une espèce animale à l'autre sont la durée unitaire d'ingestion et la quantité ingérée par grands repas.

Lorsque la longueur des brins d'ensilage est accrue, la durée unitaire d'ingestion augmente seulement chez les moutons. En revanche, la baisse de la qualité de conservation des ensilages fait diminuer nettement la quantité ingérée par grands repas chez les génisses sculement.

Lorsque les animaux reçoivent des ensilages à brins courts bien conservés, les quantités ingérées par jour (en $\mathrm{g} / \mathrm{kg} \mathrm{P0,75}$ ), les durées unitaires d'ingestion et de rumination, ainsi que la durée des grands repas sont très comparables entre moutons et génisses. La réaction comportementale des moutons à la finesse de hachage est indépendante de leur poids vif et du stade de récolte de l'ensilage.

En fin de compte, l'ensemble des résultats obtenus montrent bien :

- la moins grande sensibilté des bovins par rapport aux ovins à la finesse de hachage,

- la plus grande sensibilté des bovins à la qualité de conservation,

- l'ingestion par les moutons de quantités de matière sèche plus faibles, sous forme d'ensilage à brins courts, même très bien conservés, que de fourrage vert.
\end{abstract}

Mots clés : Ensilage, ovins, bovins, ingestibilité, comportement alimentaire. 


\section{Introduction}

La finesse de hachage des ensilages d'herbe est un des principaux facteurs de variation de leur ingestibilité par les ruminants. Les moutons ingèrent les ensilages en quantité d'autant plus élevée que ces derniers sont hachés plus finement (DuLPHY \& Demarouilly, 1973; Deswysen, Vanbelle \& Focant, 1978), la réponse étant positive jusqu'à des finesses de hachage de l'ordre de 2 à $3 \mathrm{~mm}$ (Thomas, Kelly \& WaIT, 1976). La différence des quantités ingérées entre ensilages à brins courts et longs est cependant beaucoup plus faible chez les bovins (MURDOCH, 1965 ; Lindell et al., 1970; Dulphy \& Michalet, 1975) et est même nulle dans certains essais (Duckworth \& Shirlaw, 1958 ; Wilson \& FlynN, 1974 ; de Brabander et al., 1976), la réponse étant par ailleurs nulle ou faible pour des finesses de hachage inférieures à $5 \mathrm{~cm}$ (Dulphy et al., 1983).

La qualité de conservation est également un facteur important de variation de la quantité d'ensilage ingérée (Demarouilly \& Dulphy, 1977) d'où l'influence positive nette de l'addition d'acide formique sur la quantité ingérée (SAUE \& BREIREM, 1969 ; Wilson \& Wilkins, 1973 ; Waldo, 1973 ; Dulphy \& Demarouilly, 1977).

Les réactions comparées des ovins et des bovins à ces deux facteurs de variation de la quantité d'ensilage ingérée sont mal connues (Dulphy \& Michal.eT, 1975). C'est pourquoi nous avons comparé le comportement alimentaire et mérycique de moutons et de génisses âgées de 1 an, recevant des ensilages d'herbe préparés à partir des mêmes fourrages verts et différant soit par la longueur de leurs brins, soit par leur qualité de conservation. Par ailleurs, nous avons fait varier le stade de récolte de l'ensilage pour savoir s'il modifiait l'influence de la finesse de hachage. Enfin des moutons d'âge différent ont été utilisés pour savoir si la sensibilité particulière des moutons à la finesse de hachage dépendait de leur format et de leur poids.

Les résultats concernant les fourrages verts ont déjà été présentés par ailleurs (Dulphy \& Michalet-Doreau, 1983). Ils ne seront donc cités que pour servir de témoin dans l'étude des ensilages.

\section{Matériel et méthodes}

\section{A. Fourrages}

A partir de 10 fourrages verts (tabl. 1) nous avons, de 1972 à 1978 préparé 20 ensilages d'herbe en silos-butyl de $15 \mathrm{~m}^{3}$ contenant chacun de 7 à 10 tonnes de fourrage frais. Les caractéristiques des fourrages verts et des ensilages correspondants sont rapportés dans le tableau 2. Les ensilages à brins courts (brins de 1 à $2 \mathrm{~cm}$ ) ont été récoltés avec une machine à tambour-hacheur. Ceux à brins longs $(10-15 \mathrm{~cm})$ ont été récoltés avec une machine à fléaux. Certains ensilages ont reçu 3,5 à 5 litres/tonne d'acide formique à 85 p. 100 . Le ray-grass anglais de 1978 a été ensilé à deux dates (7 juin et 28 juin). 
Tableau 1

Liste des fourrages utilisés.

Studied forages.

\begin{tabular}{|c|c|c|}
\hline $\begin{array}{l}\text { Fourrage vert } \\
\text { Green forage }\end{array}$ & $\begin{array}{l}\text { Année } \\
\text { Year }\end{array}$ & $\begin{array}{l}\text { Ensilages réalisés } \\
\text { Type of silages }\end{array}$ \\
\hline $\begin{array}{l}\text { Ray-grass anglais } \\
\text { Perennial rye-grass }\end{array}$ & 1972 & $\begin{array}{l}\text { - Brins courts }+ \text { acide formique } \\
\text { Chopped }+ \text { formic acid } \\
- \text { Brins longs }+ \text { acide formique } \\
\text { Long }+ \text { formic acid }\end{array}$ \\
\hline $\begin{array}{l}\text { Ray-grass d'Italie } \\
\text { Italian rye-grass }\end{array}$ & 1973 & $\begin{array}{l}\text { - Brins courts }+ \text { acide formique } \\
\text { Chopped }+ \text { formic acid } \\
\text { - Brins longs }+ \text { acide formique } \\
\text { Long }+ \text { formic acid }\end{array}$ \\
\hline $\begin{array}{l}\text { Fétuque élevée } . . . \cdots \\
\text { Tall fescue }\end{array}$ & 1973 & $\begin{array}{l}\text { Brins courts }+ \text { acide formique } \\
\text { Chopped }+ \text { formic acid } \\
- \text { Brins longs }+ \text { acide formique } \\
\text { Long }+ \text { formic acid }\end{array}$ \\
\hline $\begin{array}{l}\text { Ray-grass d'Italie } \\
\text { Italian rye-grass }\end{array}$ & 1974 & $\begin{array}{l}\text { - Brins courts sans conservateur } \\
\text { Chopped without preservative } \\
\text { - Brins courts }+ \text { acide formique } \\
\text { Chopped }+ \text { formic acid }\end{array}$ \\
\hline $\begin{array}{l}\text { Luzerne } \ldots . . . \\
\text { Lucerne }\end{array}$ & 1974 & $\begin{array}{l}\text { - Brins courts sans conservateur } \\
\text { Chopped without preservative } \\
\text { - Brins courts }+ \text { acide formique } \\
\text { Chopped }+ \text { formic acid }\end{array}$ \\
\hline $\begin{array}{l}\text { Ray-grass d'Italie } \\
\text { Italian rye-grass }\end{array}$ & 1975 & $\begin{array}{l}\text { - Brins courts sans conservateur } \\
\text { Chopped without preservative } \\
\text { - Brins courts }+ \text { acide formique } \\
\text { Chopped }+ \text { formic acid }\end{array}$ \\
\hline $\begin{array}{l}\text { Fétuque élevée } . . . \\
\text { Tall fescue }\end{array}$ & 1975 & $\begin{array}{l}\text { - Brins courts sans conservateur } \\
\text { Chopped without preservative } \\
\text { - Brins courts }+ \text { acide formique } \\
\text { Chopped }+ \text { formic acid }\end{array}$ \\
\hline $\begin{array}{l}\text { Ray-grass anglais } . \\
\text { Perennial rye-grass }\end{array}$ & 1976 & $\begin{array}{l}\text { - Brins courts }+ \text { acide formique } \\
\text { Chopped }+ \text { formic acid } \\
- \text { Brins longs }+ \text { acide formique } \\
\text { Long }+ \text { formic acid }\end{array}$ \\
\hline $\begin{array}{l}\text { Ray-grass anglais - Stade I . } \\
\text { Perennial rye-grass - Stage } I\end{array}$ & 1978 & $\begin{array}{l}\text { - Brins courts }+ \text { acide formique } \\
\text { Chopped }+ \text { formic acid } \\
\text { - Brins longs }+ \text { acide formique } \\
\text { Long }+ \text { formic acid }\end{array}$ \\
\hline $\begin{array}{l}\text { Ray-grass anglais - Stade II .. } \\
\text { Perennial rye-grass - Stage II }\end{array}$ & 1978 & $\begin{array}{l}\text { - Brins courts }+ \text { acide formique } \\
\text { Chopped }+ \text { formic acid } \\
\text { - Brins longs }+ \text { acide formique } \\
\text { Long }+ \text { formic acid }\end{array}$ \\
\hline
\end{tabular}


TABLeaU 2

Composition chimique, caractéristiques fermentaires et digestibilité des fourrages utilisés (moyenne et extrêmes).

\begin{tabular}{|c|c|c|c|c|}
\hline & $\begin{array}{c}\text { Teneur cn } \\
\text { matic̀re sèche } \\
\quad \% \\
\text { Dry matter } \\
\text { content } \%\end{array}$ & $\begin{array}{c}\text { Cendres } \\
\text { p. } 100 \text { M.S. } \\
\text { Ashes } \% \text { D.M. }\end{array}$ & $\begin{array}{l}\text { Matières } \\
\text { azotées } \\
\text { p. } 100 \text { M.S. } \\
\text { Crude protein } \\
\text { \% D.M. }\end{array}$ & $\begin{array}{l}\begin{array}{c}\text { Cellulose } \\
\text { brute }\end{array} \\
\text { p. } 100 \text { M.S. } \\
\text { Crude fiber } \\
\text { \% D.M. }\end{array}$ \\
\hline \multicolumn{5}{|l|}{$\mathbf{N}=6$} \\
\hline $\begin{array}{l}\text { - Fourrages verts } \ldots \ldots \\
\text { Green forages }\end{array}$ & $\begin{array}{c}17,8 \\
(16,4-19,3)\end{array}$ & $\begin{array}{c}9,6 \\
(7,9-11,4)\end{array}$ & $\begin{array}{c}11,3 \\
(7,3-13,6)\end{array}$ & $\begin{array}{c}25,8 \\
(20,7-32,7)\end{array}$ \\
\hline $\begin{array}{l}\text { Ensilages à brins courts } \\
+ \text { acide formique } \ldots . . . . \\
\text { Chopped silages with formic } \\
\text { acid }\end{array}$ & $\begin{array}{c}20,9 \\
(20,0-22,8)\end{array}$ & $\begin{array}{c}8,5 \\
(7,3-9,5)\end{array}$ & $\begin{array}{c}11,2 \\
(8,1-13,5)\end{array}$ & $\begin{array}{c}28,9 \\
(24,1-32,7)\end{array}$ \\
\hline $\begin{array}{l}\text { _ Ensilages à brins longs } \\
+ \text { acide formique } \\
\text { Long silages with formic } \\
\text { acid }\end{array}$ & $\begin{array}{c}20,9 \\
(17,5-23,2)\end{array}$ & $\begin{array}{c}13,3 \\
(10,1-18,9)\end{array}$ & $\begin{array}{c}11,1 \\
(7,9-13,6)\end{array}$ & $\begin{array}{c}27,2 \\
(22,1-34,3)\end{array}$ \\
\hline$N=4$ & & & & \\
\hline - Fourrages verts $\ldots$. & $\begin{array}{c}17,5 \\
(14,0-22,8)\end{array}$ & $\begin{array}{c}12,2 \\
(11,0-12,9)\end{array}$ & $\begin{array}{c}15,7 \\
(13,9-18,5)\end{array}$ & $\begin{array}{c}24,2 \\
(20,0-28,5)\end{array}$ \\
\hline $\begin{array}{l}\text { — Ensilages à brins courts } \\
+ \text { acide formique ......... } \\
\text { Chopped silages with formic } \\
\text { acid }\end{array}$ & $\begin{array}{c}21,3 \\
(18,2-23,3)\end{array}$ & $\begin{array}{c}10,3 \\
(9,1-11,2)\end{array}$ & $\begin{array}{c}14,6 \\
(12,4-16,9)\end{array}$ & $\begin{array}{c}25,5 \\
(20,7-28,7)\end{array}$ \\
\hline $\begin{array}{l}\text { - Ensilages à brins courts } \\
\text { sans conservateur ....... } \\
\text { Chopped silages without } \\
\text { preservative }\end{array}$ & $\begin{array}{c}19,1 \\
(14,6-23,0)\end{array}$ & $\begin{array}{c}10,5 \\
(9,7-11,4)\end{array}$ & $\begin{array}{c}16,0 \\
(14,2-18,4)\end{array}$ & $\begin{array}{c}27,6 \\
(22.8-31,0)\end{array}$ \\
\hline
\end{tabular}

\section{B. Animaux}

Tous les fourrages verts et les ensilages ont été distribués à volonté (environ 10 p. 100 de refus), comme seul aliment et en deux repas par jour, simultanément à 6 béliers castrés de race Texel, âgés de 2,5 ans et pesant $60 \mathrm{~kg}$ en moyenne, maintenus en cage à métabolisme et à 5 génisses de race Frisonne, âgées de 1 an et pesant 
Chemical composition, fermentation characteristics and digestibility of the studied forages (mean, minimum and maximum).

\begin{tabular}{|c|c|c|c|c|c|}
\hline \multirow{2}{*}{ pH } & \multicolumn{3}{|c|}{$\begin{array}{l}\text { Acides organiques } \mathrm{g} / \mathrm{kg} \text { M.S. } \\
\text { Organic acids } \mathrm{g} / \mathrm{kg} \text { D.M. }\end{array}$} & \multirow{2}{*}{$\begin{array}{c}\mathrm{N}-\mathrm{NH} \\
\% \\
\mathrm{~N} \text { total } \\
\mathrm{NH}_{3}-\mathrm{N} \\
\% \\
\text { total } \mathrm{N}\end{array}$} & \multirow{2}{*}{$\begin{array}{c}\text { Digestibilité } \\
\text { de la matière } \\
\text { organique } \\
\text { Digestibility } \\
\text { of organic } \\
\text { matter }\end{array}$} \\
\hline & $\begin{array}{l}\text { lactique } \\
\text { lactic }\end{array}$ & $\begin{array}{l}\text { acétique } \\
\text { acetic }\end{array}$ & $\begin{array}{c}\text { butyrique } \\
\text { butyric }\end{array}$ & & \\
\hline- & - & - & - & - & $\begin{array}{c}70,5 \\
(61,9-77,9)\end{array}$ \\
\hline $\begin{array}{c}3,89 \\
(3,77-4,10)\end{array}$ & $\begin{array}{c}53 \\
(44-69)\end{array}$ & $\begin{array}{c}17 \\
(11-22)\end{array}$ & $\begin{array}{c}0,2 \\
(0,0-1,0)\end{array}$ & $\begin{array}{c}6,4 \\
(4,7-8,7)\end{array}$ & $\begin{array}{c}69,3 \\
(63,0-77,0)\end{array}$ \\
\hline $\begin{array}{c}4,10 \\
(3,95-4,55)\end{array}$ & $\begin{array}{c}58 \\
(24-85)\end{array}$ & $\begin{array}{c}25 \\
\left(13^{-40)}\right.\end{array}$ & $\begin{array}{c}3,2 \\
(0,0-7,0)\end{array}$ & $\begin{array}{c}8,5 \\
(6,6-15,7)\end{array}$ & $\begin{array}{c}72,1 \\
(64,4-81,6)\end{array}$ \\
\hline- & - & - & - & - & $\begin{array}{c}71,1 \\
(62,8-81,3)\end{array}$ \\
\hline $\begin{array}{c}3,88 \\
(3,65-4,22)\end{array}$ & $\begin{array}{c}60 \\
(42-79)\end{array}$ & $\begin{array}{c}20 \\
(11-29)\end{array}$ & 0,0 & $\begin{array}{c}5,8 \\
(3,5-7,8)\end{array}$ & $\begin{array}{c}70,2 \\
(59,9-79,0)\end{array}$ \\
\hline $\begin{array}{c}4,49 \\
(4,10-4,75)\end{array}$ & $\begin{array}{c}48 \\
(29-86)\end{array}$ & $\begin{array}{c}70 \\
(47-99)\end{array}$ & 0,0 & $\begin{array}{c}11,3 \\
(6,2-15,2)\end{array}$ & $\begin{array}{c}70,9 \\
(61,3-79,6)\end{array}$ \\
\hline
\end{tabular}

$320 \mathrm{~kg}$ en moyenne. En 1978, 12 béliers ont été employés, 6 de 1,5 an pesant $45 \mathrm{~kg}$ et 6 de 3 à 4 ans pesant $75 \mathrm{~kg}$ en moyenne. Les animaux utilisés pour les mesures sur les fourrages verts au printemps et sur les ensilages correspondants au cours de l'hiver suivant, ont été choisis avec un âge et un poids aussi comparables que possible. 


\section{Mesures}

En été les fourrages étaient récoltés chaque jour et chacun a été distribué pendant 16 jours (sous forme hachée aux moutons : environ $3 \mathrm{~cm}$, et sous forme longue aux génisses), les 6 derniers jours constituant la période expérimentale. C'est au milieu de cette période qu'étaient préparés les deux ensilages correspondant à ce fourrage vert. En hiver les ensilages étaient distribués chacun pendant 21 jours, l'un après l'autre, les 6 derniers jours constituant la période expérimentale.

Les quantités individuelles journalières de matière sèche ingérée ont été mesurées par pesée et détermination de la teneur en matière sèche du fourrage offert à chacune des deux distributions et des refus enlevés le lendemain matin avant la distribution du premier repas de la journée. On a, en outre, mesuré — sauf en 1978 - la quantité ingérée lors des deux grands repas journaliers (repas suivant chacune des deux distributions). Les manifestations du comportement alimentaire et mérycique des animaux ont été enregistrées chaque jour, lors des périodes expérimentales, selon la technique de Ruckebusch (1963). La définition des paramètres que nous avons utilisés pour décrire ce comportement a été donnée par DulPHY \& Demarouilly (1974). Nous avons en outre appelé «temps de latence» le temps écoulé entre la fin des deux grands repas et le début de la première période de rumination leur faisant suite. Les moutons, grâce à la mesure des quantités de fèces qu'ils ont émises, ont servi à mesurer la digestibilité de tous les fourrages utilisés (tabl. 2).

\section{Analyses}

La teneur en matière sèche des fourrages a été déterminée par séchage dans une étuve ventilée à $80^{\circ} \mathrm{C}$ pendant 24 heures. Pour les ensilages, cette teneur a été corrigée selon la méthode de Dulphy, Demarouilly \& Henry (1975). Sur les échantillons secs, nous avons déterminé les teneurs en cendres et en cellulose brute, la teneur en azote de l'ensilage étant, en revanche, déterminée sur l'ensilage frais.

\section{Résultats}

Les résultats moyens figurent, pour chaque paramètre analysé, dans les tableaux 3,4 et 5 .

Dans le tableau 3 se trouvent, pour les moutons et les génisses, les résultats relatifs aux comparaisons fourrage vert-ensilage à brins courts additionné d'acide formique, soit 8 comparaisons puisque en 1978 les fourrages verts n'ont pas été distribués aux génisses.

Dans le tableau 4 se trouvent les résultats relatifs aux 6 comparaisons ensilage à brins courts - ensilage à brins longs et dans le tableau 5 ceux relatifs aux 4 comparaisons entre ensilages avec et sans acide formique. 
Enfin, dans le tableau 6, les résultats ont été présentés de façon groupée en affectant l'indice 100 au fourrage vert, puis à l'ensilage à brins courts avec acide formique.

\section{A. Comparaison des différents paramètres et interaction avec la technique d'ensilage}

\section{Quantités ingérées}

Les quantités de matière sèche ingérées de chaque ensilage, par rapport au fourrage vert initial, sont reportées dans les figures 1 et 2 , respectivement pour les moutons et les génisses. Globalement les génisses ont ingéré, en $\mathrm{g} / \mathrm{kg} \mathrm{P}^{0.75}$, nettement plus que les moutons.

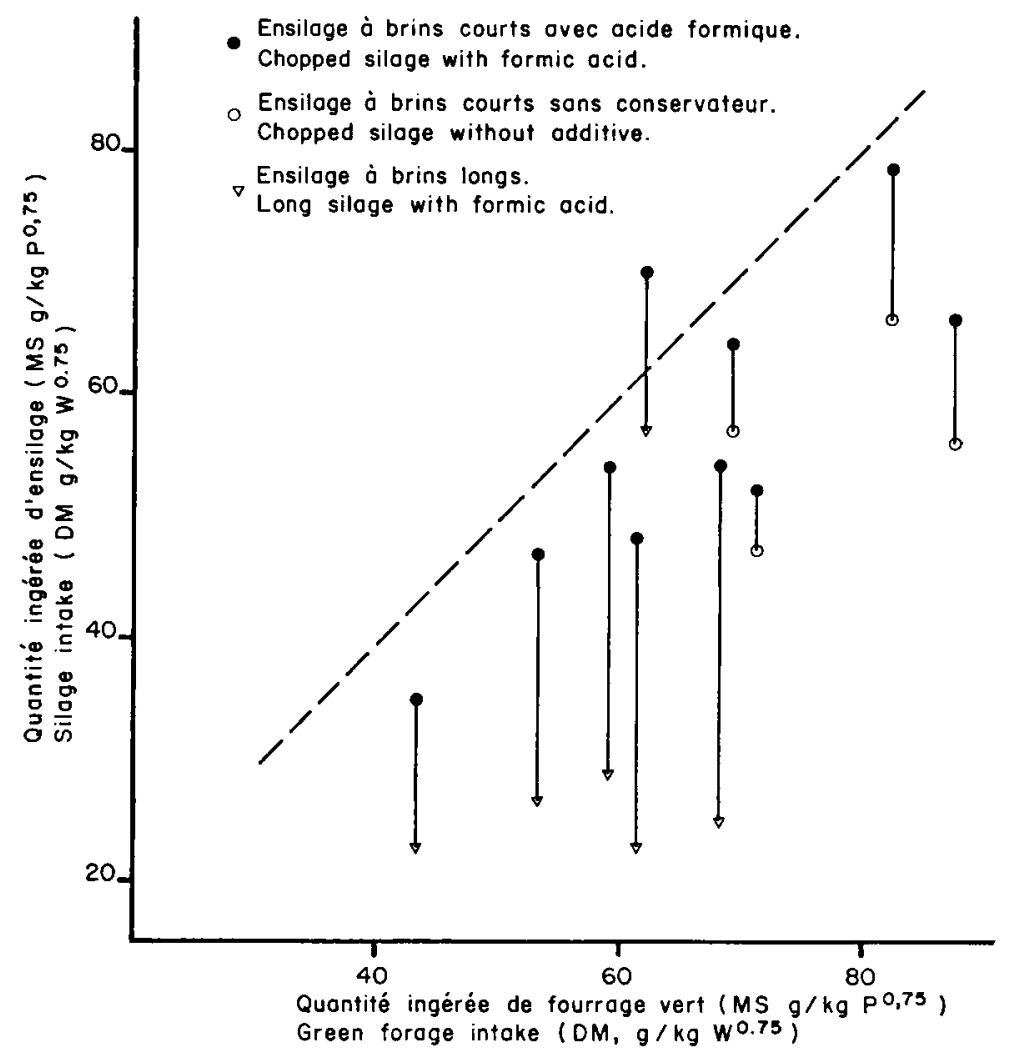

FIG. 1

Relation entre quantité d'ensilage et quantité de fourrage vert ingérée chez le mouton. Relationship between silage and green forage intake on sheep. 
氙

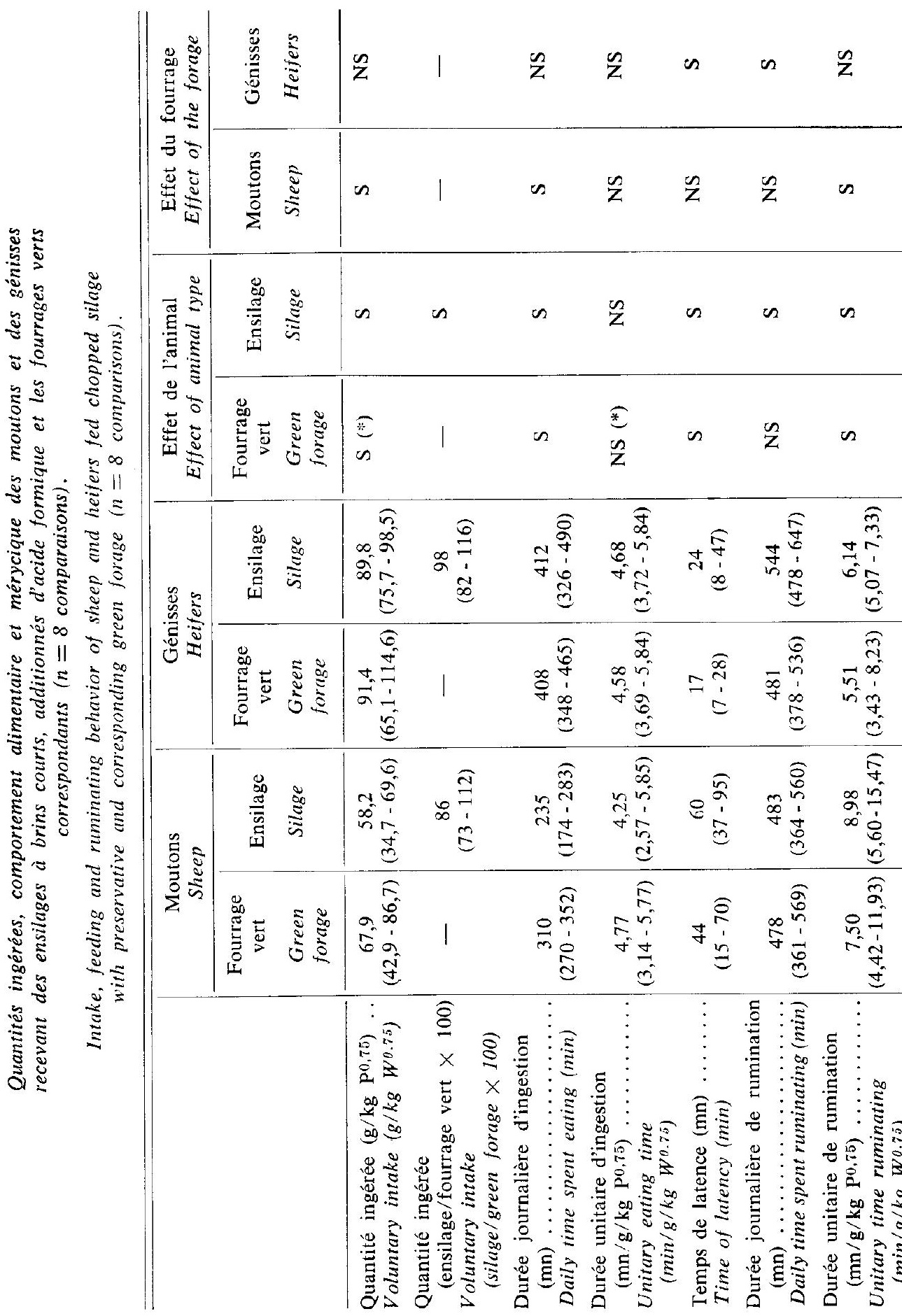




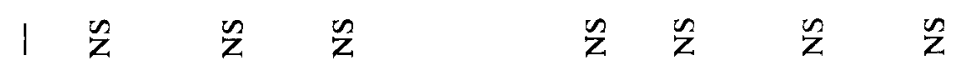

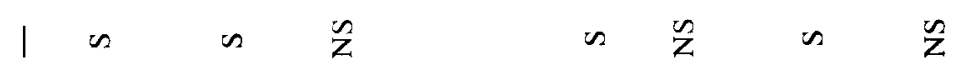

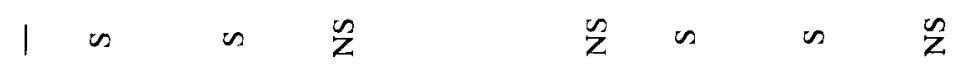

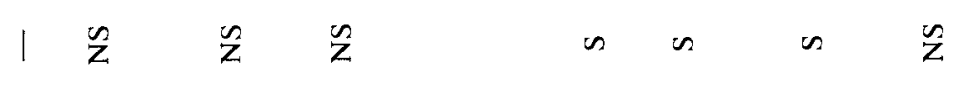

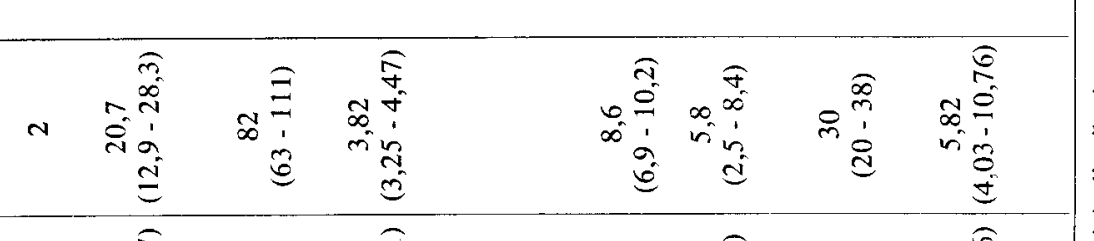

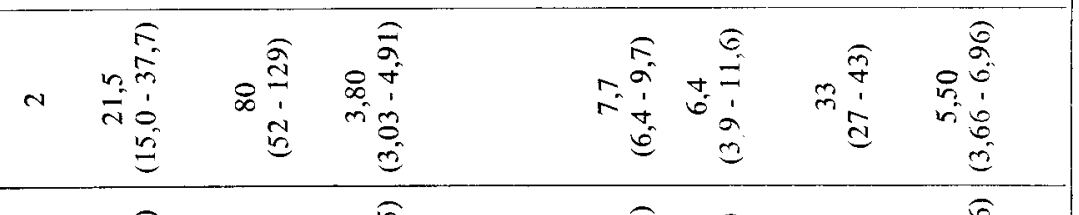

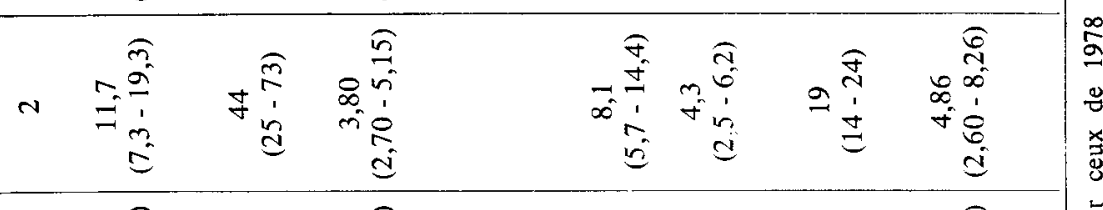

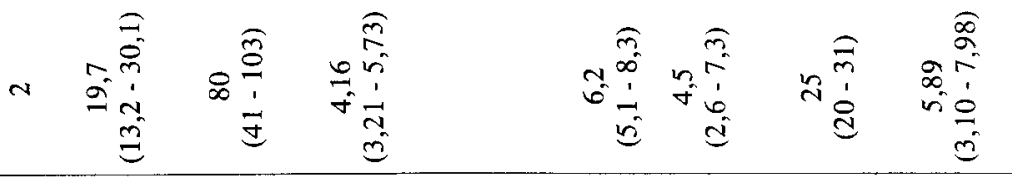

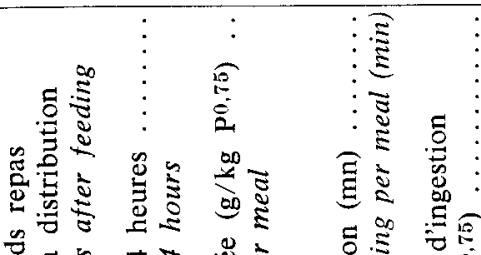

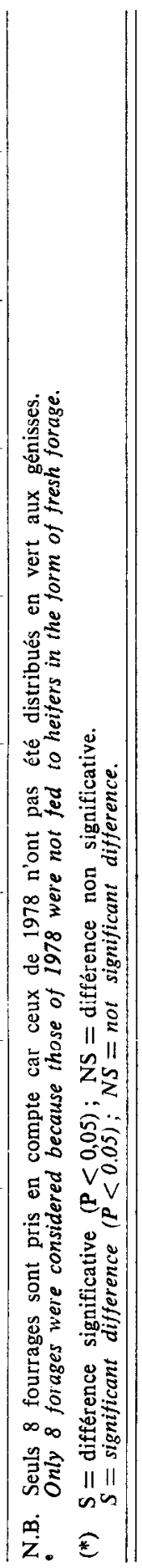

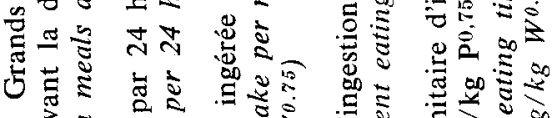

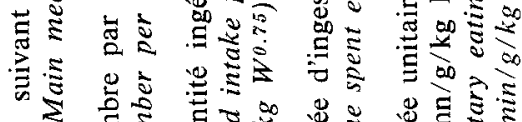

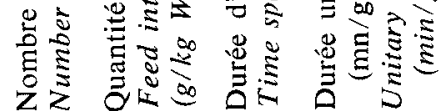

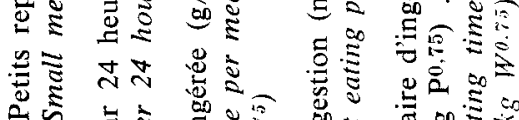

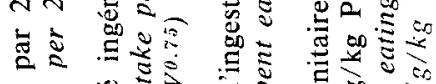

गे

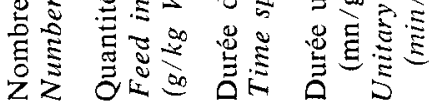


嵩

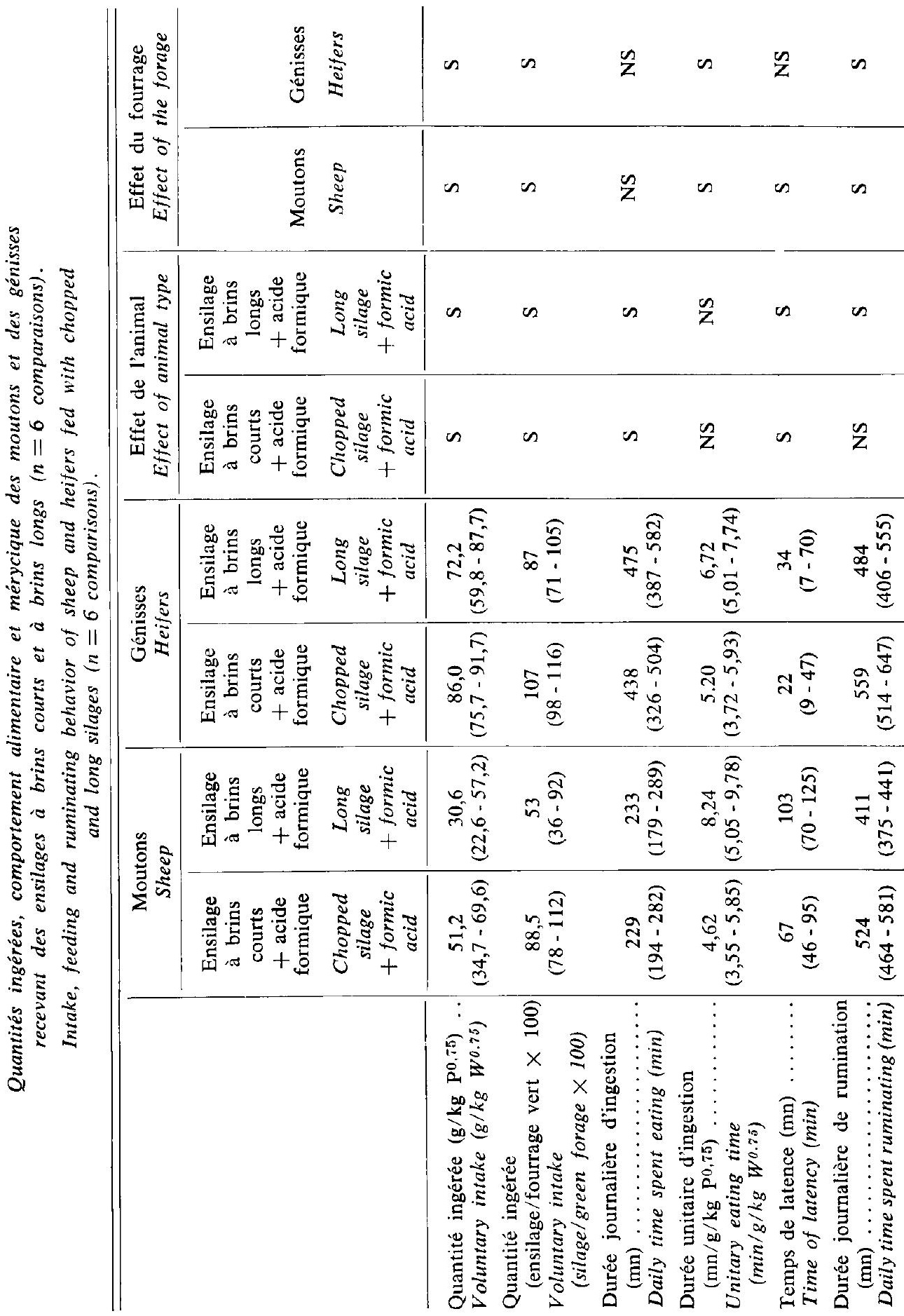




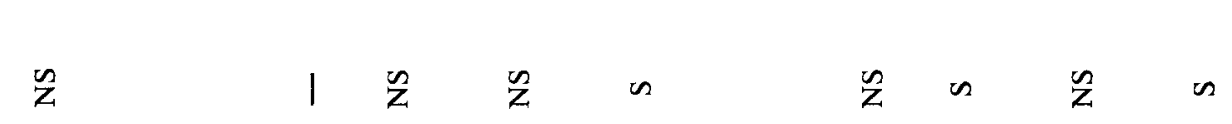

\begin{tabular}{|c|c|c|c|c|c|c|c|c|c|c|}
\hline$n$ & & 1 & $n$ & $\tilde{Z}$ & n & & $\tilde{Z}$ & $\tilde{z}$ & $\tilde{z}$ & $\sim$ \\
\hline$\infty$ & & 1 & $n$ & $n$ & $n$ & & $n$ & Z & $\tilde{z}$ & n \\
\hline ns & & 1 & $n$ & $\infty$ & 乙 & & $\check{Z}$ & Z & $n$ & $\widetilde{\Sigma}$ \\
\hline 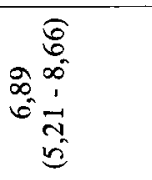 & & $\mathrm{N}$ & 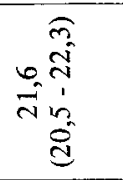 & 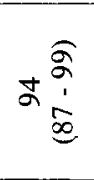 & 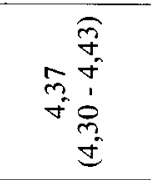 & & $\begin{array}{c}0 \\
\sigma= \\
\sigma \\
0 \\
0 \\
0\end{array}$ & 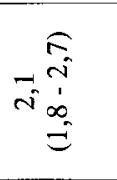 & 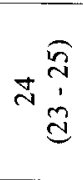 & 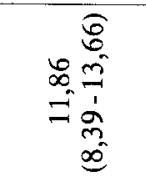 \\
\hline 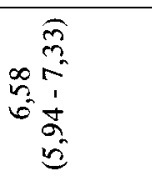 & & $N$ & 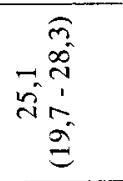 & 志 & 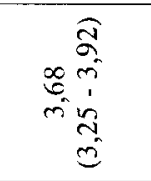 & & 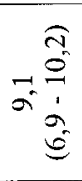 & 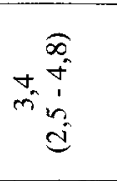 & $\stackrel{\overparen{I}}{\stackrel{\overbrace{}}{1}}$ & 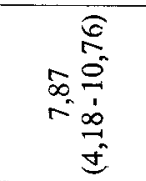 \\
\hline 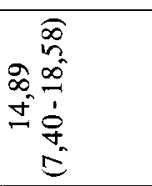 & & $N$ & 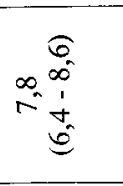 & $\begin{array}{r}\widehat{\widehat{\vartheta}} \\
-\overrightarrow{1} \\
\infty \\
0 \\
0\end{array}$ & 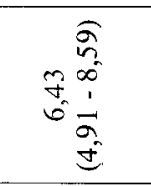 & & 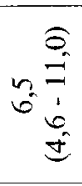 & $\begin{array}{r}\text { कo } \\
m \stackrel{m}{m} \\
\stackrel{n}{=} \\
=\end{array}$ & 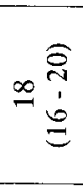 & 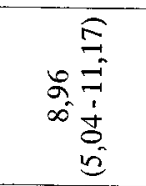 \\
\hline 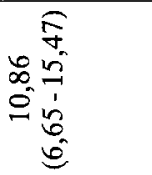 & & $N$ & 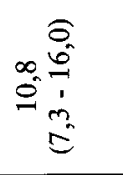 & $\underset{\vec{n}}{\tilde{a}}$ & 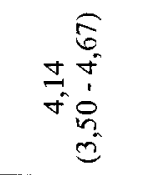 & & $\frac{\sqrt{d}}{\sqrt[d]{ \pm}}$ & 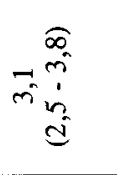 & 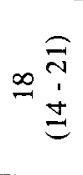 & 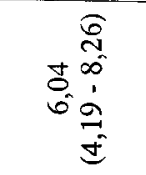 \\
\hline 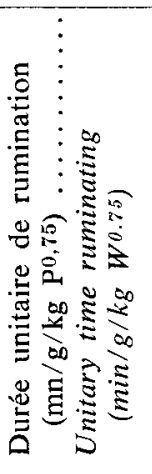 & 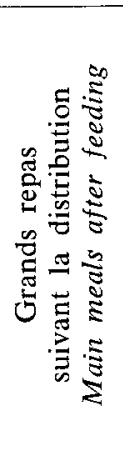 & 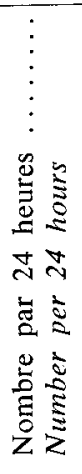 & 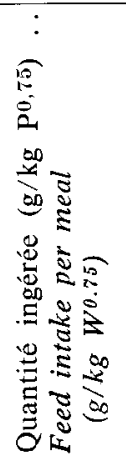 & 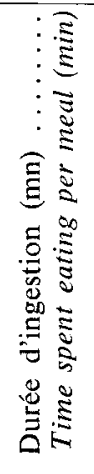 & 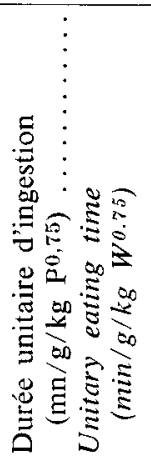 & 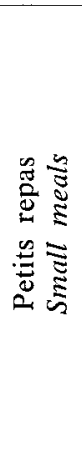 & 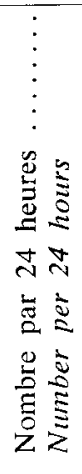 & 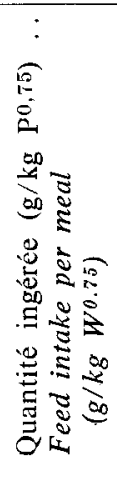 & 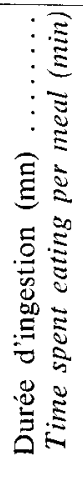 & 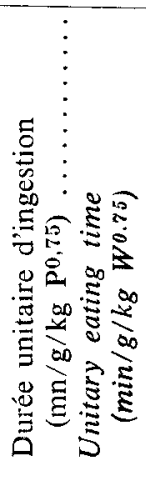 \\
\hline
\end{tabular}




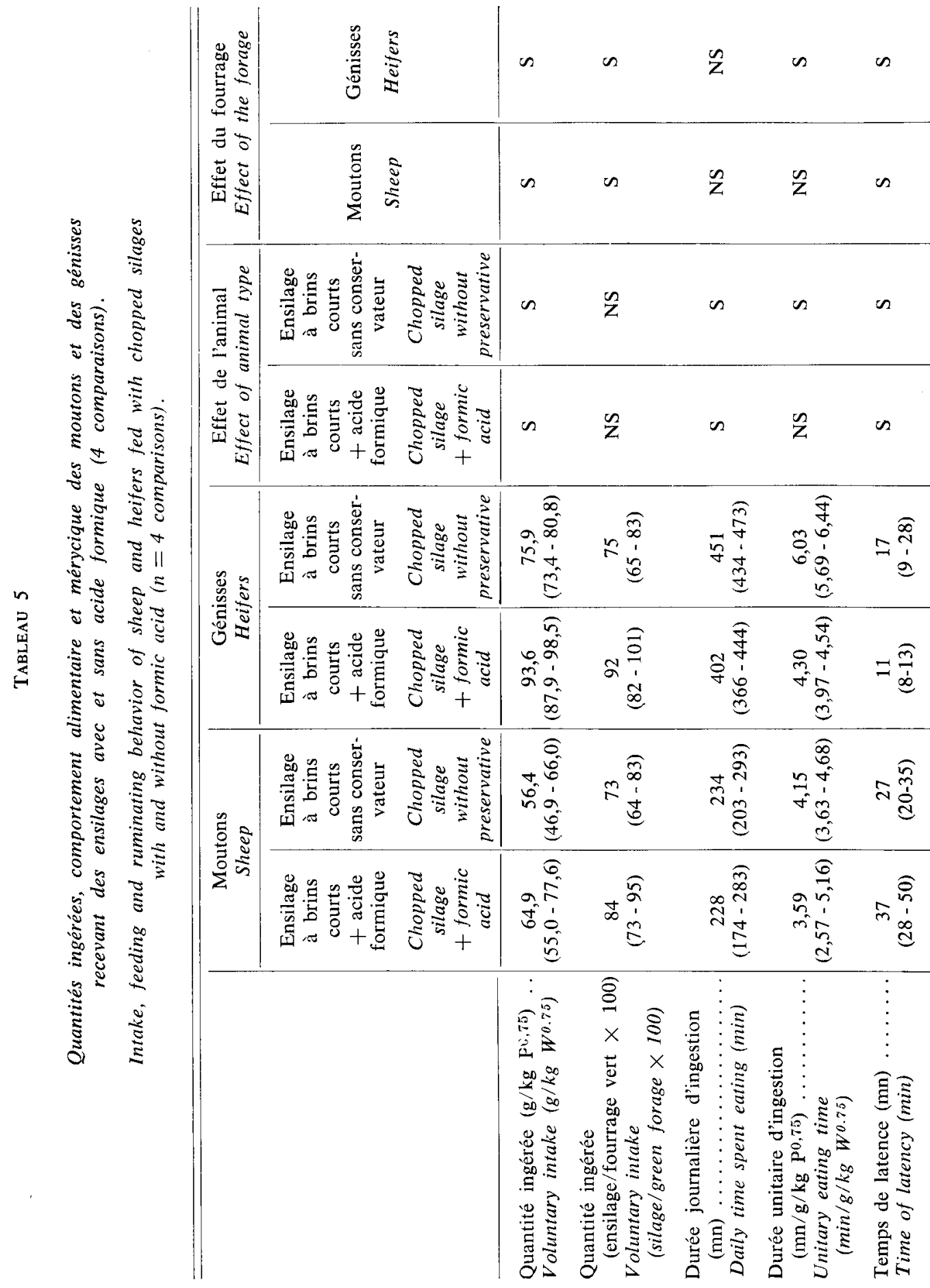




\begin{tabular}{|c|c|c|c|c|c|c|c|c|c|}
\hline in & $\infty$ & 1 & $n$ & $\tilde{z}$ & $\bar{z}$ & $\tilde{z}$ & $\tilde{z}$ & $\tilde{z}$ & $n$ \\
\hline$\tilde{z}$ & z & 1 & $\tilde{z}$ & $\tilde{z}$ & $\tilde{z}$ & $\tilde{z}$ & $\tilde{z}$ & $\tilde{z}$ & $\tilde{z}$ \\
\hline$\frac{n}{z}$ & $\tilde{z}$ & 1 & $\tilde{z}$ & $n$ & $z$ & $\underline{n}$ & $\bar{z}$ & on & 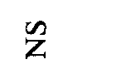 \\
\hline$\tilde{z}$ & $\tilde{z}$ & 1 & $\infty$ & $n$ & $\sum_{z}^{n}$ & $\tilde{z}$ & in & $\infty$ & $n$ \\
\hline & 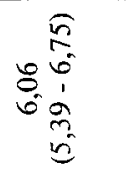 & N & 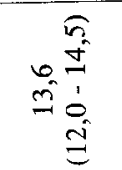 & 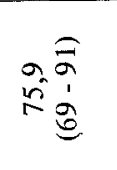 & 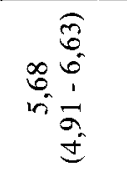 & & $\begin{array}{c}\hat{6} \\
0 \\
0 \\
0 \\
0 \\
0 \\
0\end{array}$ & 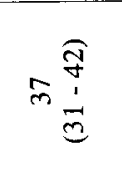 & 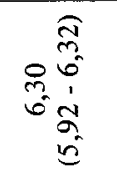 \\
\hline i్ & 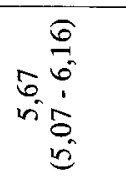 & $\mathrm{N}$ & 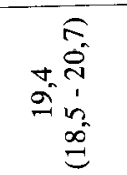 & 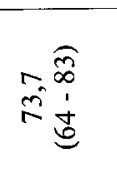 & 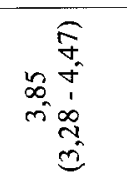 & & 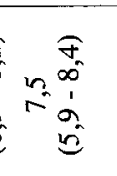 & 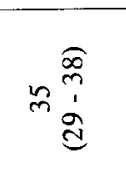 & 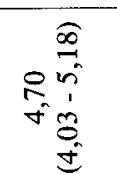 \\
\hline & 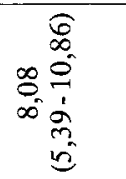 & N & 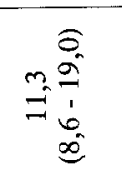 & 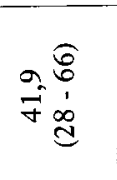 & 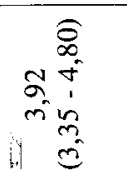 & & 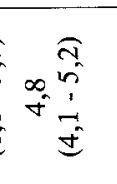 & 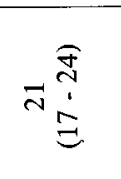 & 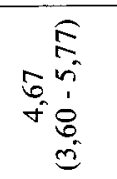 \\
\hline $\begin{array}{l}3 \\
7\end{array}$ & 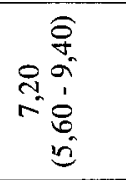 & $\sim$ & 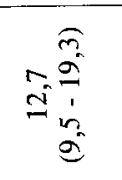 & $\underset{\sigma}{\widehat{\sigma}}$ & 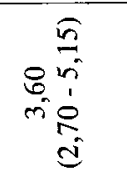 & ?ר? & 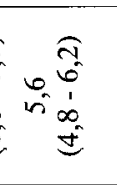 & 尺 & 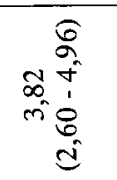 \\
\hline 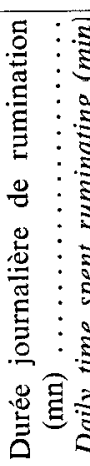 & 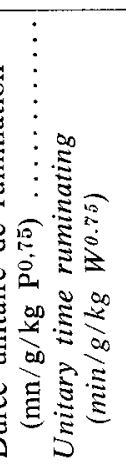 & 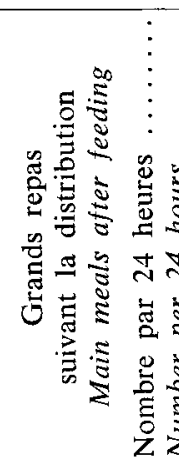 & 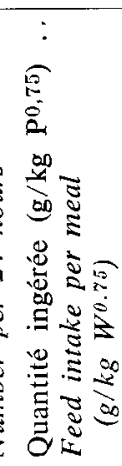 & 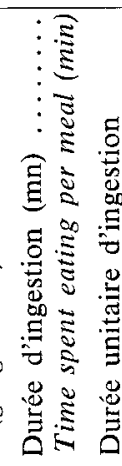 & 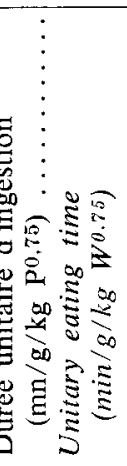 & 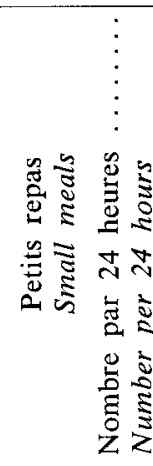 & 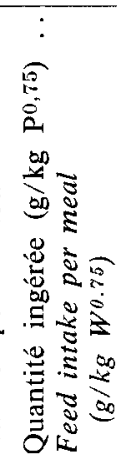 & 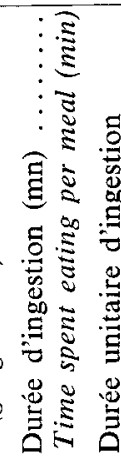 & 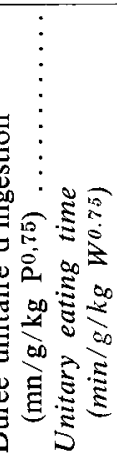 \\
\hline
\end{tabular}




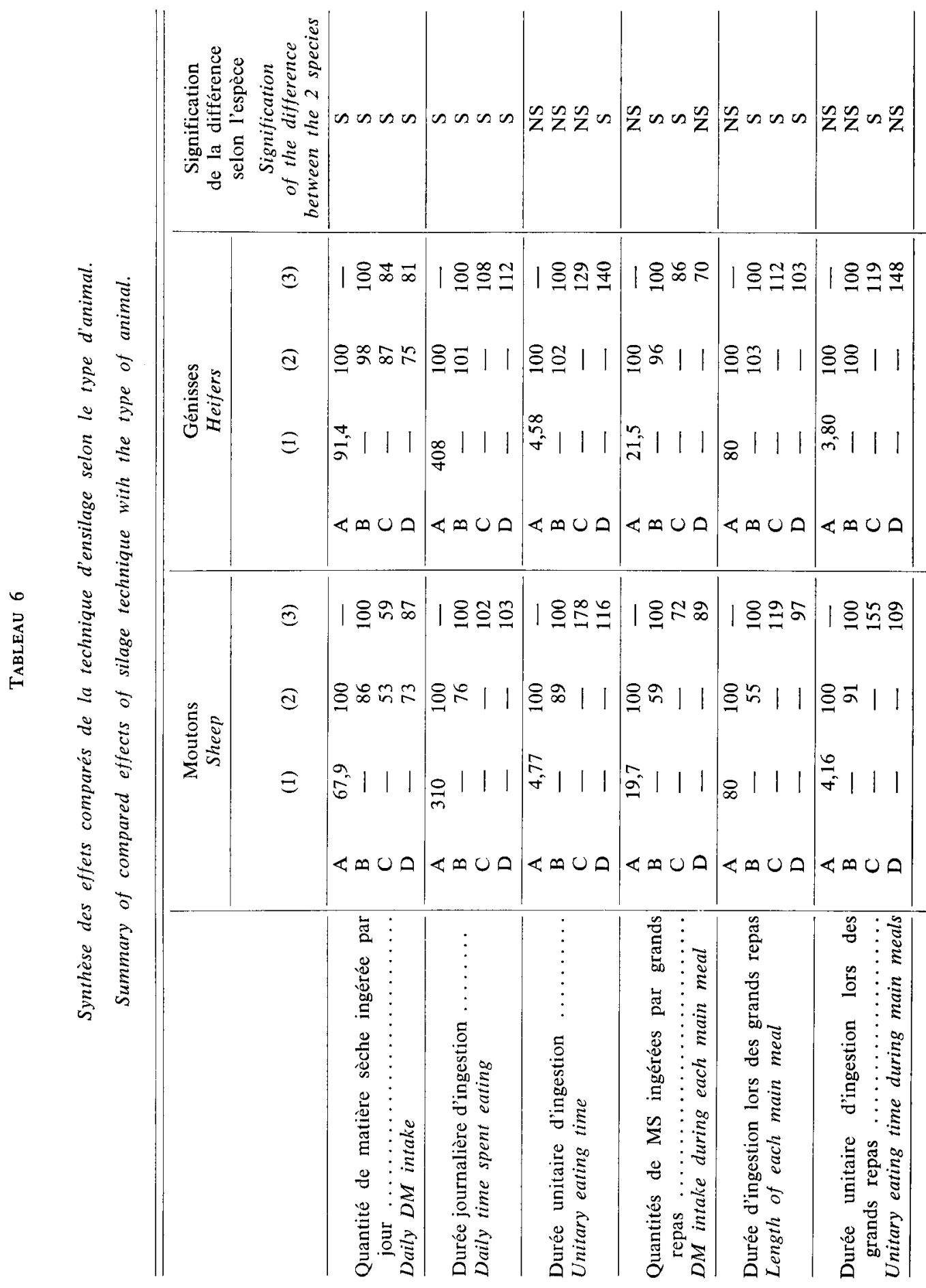




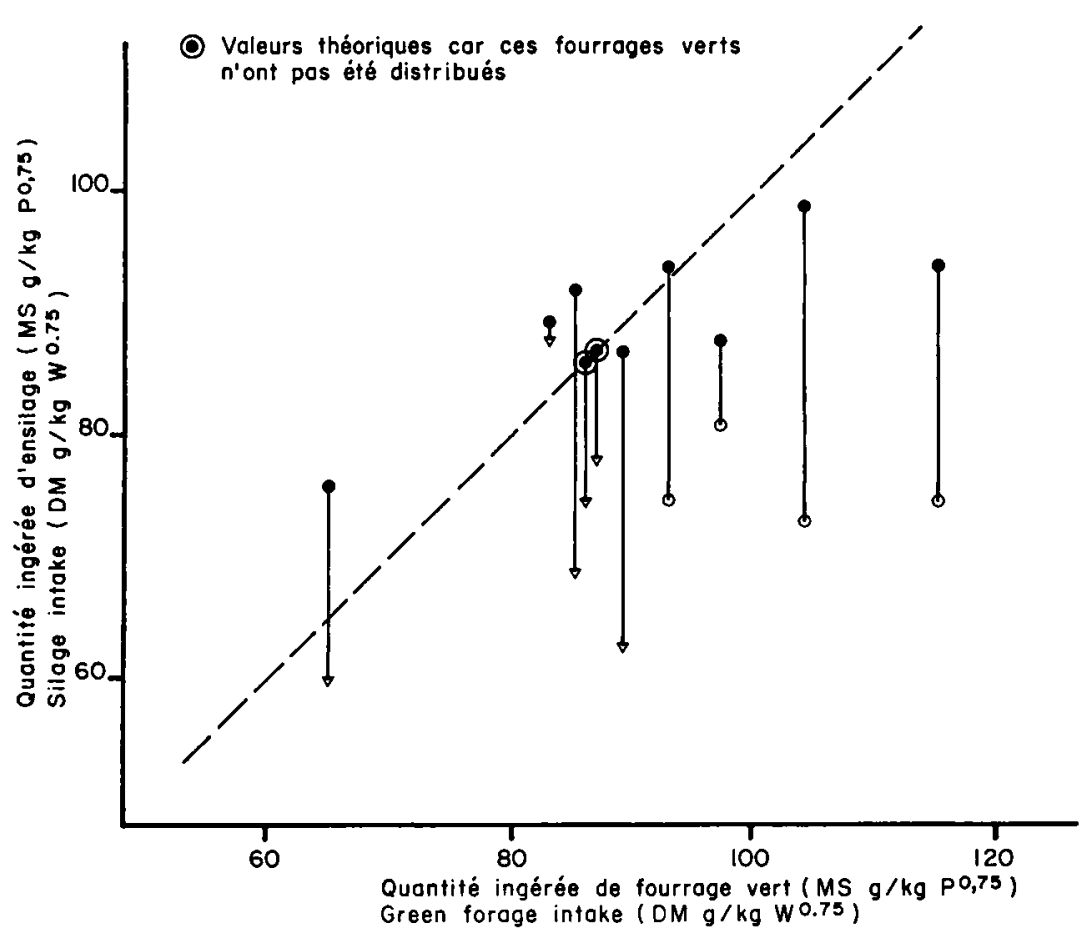

FIG. 2

Relation entre quantité d'ensilage et quantité de fourrage vert ingérée chez la génisse.

Relationship between silage and green forage intake in heifers.

L'allongement des brins ou la suppression du conservateur s'est traduit par une diminution importante de la quantité d'ensilage ingérée; cette diminution a été cependant très variable d'une comparaison à l'autre, notamment en cas d'allongement des brins. En effet, exprimée en p. 100 de la quantité d'ensilage à brins courts avec conservateur ingérée, celle d'ensilage à brins longs avec conservateur a varié de 46 à 82 p. 100 chez le mouton et de 73 à 98 p. 100 chez la génisse et celle d'ensilage à brins courts sans conservateur a varié de 84 à 90 p. 100 chez le mouton et de 75 à 92 p. 100 chez la génisse.

Il n'y a pas eu d'influence du stade de récolte sur les écarts relatifs entre ensilages à brins longs, à brins courts et fourrages verts (tabl. 9).

La quantité ingérée en $\mathrm{g}$ de $\mathrm{MS} / \mathrm{kg} \mathrm{P}^{0,75}$ (QI par le mouton $\mathrm{M}$ ou la génisse $\mathrm{G}$ ) d'ensilage à brins courts avec acide formique (BCAF) a été liée étroitement à celle du fourrage vert initial (FV) :

$$
\begin{aligned}
& \mathrm{QI}_{\mathrm{M}} \mathrm{BCAF}=0,777 \mathrm{QI}_{\mathrm{M}} \mathrm{FV}+5,8( \pm 8,2 ; \mathrm{r}=0,794 ; \mathrm{n}=10) \\
& \mathrm{QI}_{\mathrm{G}} \mathrm{BCAF}=0,379 \mathrm{QI}_{\mathrm{G}} \mathrm{FV}+55,2( \pm 4,2 ; \mathrm{r}=0,820 ; \mathrm{n}=10)
\end{aligned}
$$


De plus, pour ces ensilages, les quantités ingérées par les moutons et les génisses ont été étroitement liées (fig. 3).

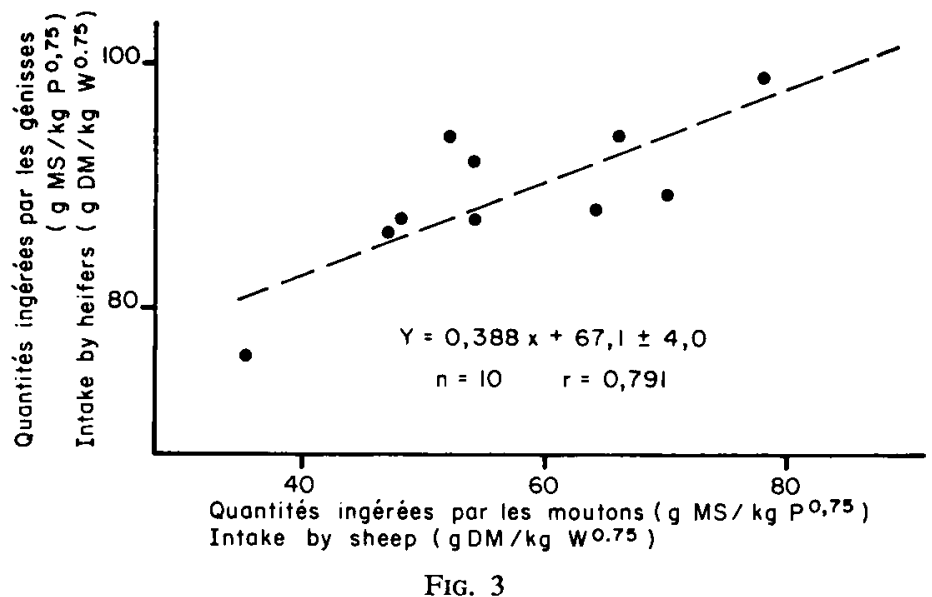

Relation entre les quantités ingérées d'ensilage à brins courts ( + acide formique) par les moutons et les génisses.

Relationship between chopped silage ( + formic acid) intake in sheep and heifers.

\section{Durée journalière d'ingestion}

Les génisses ont passé en moyenne plus de temps à ingérer que les moutons et les différences ont augmenté lorsqu'on est passé des fourrages verts aux ensilages. Par contre, pour aucune espèce animale, ni l'effet de la longueur des brins, ni celui de l'acide formique n'ont été significatifs, bien que chez les génisses, l'ensilage à brins courts avec conservateur ait été ingéré pendant un temps inférieur à ceux des deux autres types d'ensilage.

\section{Durée unitaire d'ingestion}

Les durées unitaires d'ingestion ont été comparables pour les moutons et les génisses, sauf avec les ensilages à brins courts sans conservateur. La diminution de quantité ingérée observée chez les moutons entre le fourrage vert et l'ensilage à brins courts avec conservateur a donc résulté essentiellement de celle de la durée journalière d'ingestion, puisque la durée unitaire d'ingestion de l'ensilage a été plus faible (ce qui correspond à une ingestion plus rapide) que celle du fourrage vert.

L'augmentation de la longueur des brins a entraîné une augmentation très importante de la durée unitaire d'ingestion, notamment chez le mouton. De même, la suppression de l'acide formique a entraîné une augmentation importante de cette durée unitaire mais, cette fois, surtout chez la génisse. La diminution de la quantité d'ensilage ingérée consécutive à l'allongement des brins ou à la suppression de l'acide formique a donc résulté essentiellement de l'augmentation de la durée unitaire 
d'ingestion, puisque la durée journalière d'ingestion n'a pas été modifiée chez le mouton et a même augmenté chez la génisse.

Quel que soit le type d'ensilage (ENS), il a d'ailleurs existé des liaisons étroites entre la quantité ingérée (QI) et la durée unitaire d'ingestion (DUI) :

- pour les moutons

$$
\mathrm{QI}_{\mathrm{M}} \mathrm{ENS}=-6,63 \mathrm{DUI}+84,7( \pm 7,8 ; \mathrm{r}=0,890 ; \mathrm{n}=20)
$$

- pour les génisses

$$
\mathrm{QI}_{\mathrm{G}} \mathrm{ENS}=-7,19 \text { DUI }+121,8( \pm 6,7 ; \mathrm{r}=0,789 ; \mathrm{n}=20)
$$

Pour les ensilages à brins courts avec conservateur (BCAF) on a eu :

- pour les moutons

$$
\mathrm{QI}_{\mathrm{M}} \mathrm{BCAF}=-8,42 \mathrm{DUI}+92,2( \pm 9,0 ; \mathrm{r}=0,741 ; \mathrm{n}=10)
$$

- pour les génisses

$$
\mathrm{QI}_{G} \mathrm{BCAF}=-5,24 \mathrm{DUI}+114,4( \pm 4,8 ; \mathrm{r}=0,681 ; \mathrm{n}=10)
$$

\section{Quantité de matière sèche ingérée lors des grands repas}

Lors des grands repas les moutons et les génisses ont ingéré, en $\mathrm{g} / \mathrm{kg} \mathrm{P}^{0.7 \pi}$, des quantités comparables de fourrage vert. Il en a été de même pour les quantités ingérées d'ensilage à brins courts sans conservateur. Par contre, les génisses ont ingéré nettement plus les ensilages avec conservateur, qu'ils soient à brins longs ou à brins courts.

Globalement, avec les ensilages à brins courts avec conservateur, les moutons ont eu des quantités ingérées faibles par rapport aux fourrages verts. L'augmentation de la longueur des brins a eu un effet négatif significatif chez les moutons, mais pas chez les génisses alors que l'inverse a été observé lors de la suppression de l'acide formique.

Pour les ensilages à brins courts avec conservateur, la différence de quantité ingérée lors de ces grands repas par rapport aux fourrages verts a été due essentiellement à une diminution très forte de la durée des grands repas chez les moutons (tabl. 3). En effet, les durées unitaires d'ingestion des ensilages à brins courts sans conservateur et des fourrages verts ont été comparables chez les moutons et les génisses.

L'augmentation de la longueur des brins n'a pas modifié la durée des grands repas, ni chez les moutons, ni chez les génisses. Par contre, les durées unitaires d'ingestion correspondantes ont augmenté pour les deux espèces.

On retrouve, au niveau des grands repas, pour la durée unitaire d'ingestion, l'effet fortement positif de la longueur des brins pour les moutons et de la suppression du conservateur pour les génisses.

La durée d'ingestion telle que nous l'avons mesurée ne donne pas une idée exacte de la façon dont l'animal mastique car il ne mastique pas de façon continue au cours d'un repas. En effet, une partie du temps est consacrée à la recherche ou au tri du fourrage. Sur les ensilages de 1973, nous avons étudié de façon détaillée 
les activités des animaux au cours des dix premières minutes du repas suivant la distribution du soir, en faisant dérouler rapidement la bande d'enregistrement (50 $\mathrm{mm} / \mathrm{mn}$ contre 2 habituellement). Nous avons pu ainsi distinguer, pendant ces dix minutes :

- des périodes sans mastication qui ont été assimilées à un temps de recherche : 36 p. 100 de la durée d'observation pour les moutons et seulement 14 p. 100 pour les génisses;

- des périodes avec mastication, au cours desquelles nous avons mesuré le nombre de coups de mâchoire par minute, soit 120 pour les moutons et 92 pour les génisses. Certes, ces valeurs ne sont qu'indicatives car le temps passé à la recherche et le nombre de coups de mâchoire par minute varient au cours du repas (GILL, Campling \& Westgarth, 1966 ; Deswysen \& Ehrlein, 1981) et nos mesures n'ont été effectuées qu'en début de repas. Malgré tout, on peut comparer dans ces conditions les fourrages et les espèces animales. Ainsi, en ramenant le nombre de coups de mâchoire à la quantité de fourrage ingérée au cours d'un grand repas, on trouve (tabl. 7) :

\section{TAeleau 7}

Mastication lors de l'ingestion d'un grand repas (ensilages de 1973).

Chewing during eating of main meal (silages of 1973).

\begin{tabular}{|c|c|c|c|c|}
\hline & $\begin{array}{c}\text { Durée } \\
\text { d'ingestion } \\
\text { Time spent } \\
\text { eating (min) }\end{array}$ & $\begin{array}{c}\text { Temps } \\
\text { de recherche } \\
\text { de l'aliment } \\
(\%) \\
\text { Time spent } \\
\text { searching } \\
\text { the feedstuffs } \\
(\%)\end{array}$ & $\begin{array}{c}\text { Nombre } \\
\text { de coups de } \\
\text { mâchoire/mn } \\
\text { Number of } \\
\text { chewing/min }\end{array}$ & $\begin{array}{c}\text { Nombre } \\
\text { de coups de } \\
\text { mâchoire/g } \\
\text { de fourrage } \\
\text { ingéré } \\
\text { Number of } \\
\text { chewing/g } \\
\text { of forage } \\
\text { intake }\end{array}$ \\
\hline \multicolumn{5}{|l|}{$\begin{array}{l}\text { Moutons } \\
\text { Sheep }\end{array}$} \\
\hline $\begin{array}{l}\text { - Ensilage à brins longs } \\
\text { Long silage }\end{array}$ & $a 55 \pm 6$ & 37 & $116 \pm 13$ & $a_{28,5}$ \\
\hline $\begin{array}{l}\text { Ensilage à brins courts } \\
\text { Chopped silage }\end{array}$ & $a 56 \pm 17$ & 36 & $\mathrm{a} 124 \pm 10$ & b 18,3 \\
\hline \multicolumn{5}{|l|}{$\begin{array}{l}\text { Génisses } \\
\text { Heifers }\end{array}$} \\
\hline $\begin{array}{l}\text { - Ensilage à brins longs } \\
\text { Long silage }\end{array}$ & a97 \pm 5 & 14 & $a 8=8$ & a 4,4 \\
\hline $\begin{array}{l}\text { - Ensilage à brins courts . } \\
\text { Chopped silage }\end{array}$ & a $109 \pm 8$ & 15 & a $97 \pm 11$ & $\mathrm{a} 4,2$ \\
\hline
\end{tabular}

(Les valeurs affectées de lettres différentes sont significativement différentes à $\mathbf{P}<0,05$ Analyse de variance à 1 voie de classification.)

(Values with different letters have statistical difference at $P<0.05$. Analysis of variance for a one way classification.) 
- que les moutons ont mastiqué beaucoup plus leurs aliments que les génisses (respectivement 23,4 coups de mâchoire par g de MS de fourrage ingéré, contre 4,3 chez les génisses) ;

- que le nombre de coups de mâchoire est passé, chez les moutons, de 18,3/g pour les ensilages à brins courts, à $28,5 / \mathrm{g}$ pour les ensilages à brins longs, alors que la variation n'a pas été significative chez les génisses (respectivement 4,2 et 4,4 g).

\section{Nombre de petits repas}

Les génisses ont toujours effectué plus de petits repas que les moutons mais les différences n'ont été significatives que pour les fourrages verts et les ensilages à brins longs. L'augmentation de la longueur des brins a entraîné une baisse, quoique non significative, de ce nombre de repas chez les moutons, mais pas chez les génisses. La suppression du conservateur n'a pas eu d'effet significatif chez aucune des espèces.

\section{Quantité ingérée par petits repas}

En général les génisses ont ingéré plus, en $\mathrm{g}$ de $\mathrm{MS} / \mathrm{kg} \mathrm{P}^{0,75}$, que les moutons lors de chacun des petits repas. Ceci a été surtout marqué pour les fourrages verts et les ensilages à brins courts avec conservateur.

L'augmentation de la longueur des brins d'ensilage a entraîné une baisse systématique de la quantité ingérée par petits repas, plus nette chez les génisses que chez les moutons. Il en a été de même après la suppression du conservateur.

Enfin, lors de ces petits repas, la durée unitaire d'ingestion des ensilages à brins courts avec conservateur a été plus faible (ingestion plus rapide) que celle des fourrages verts chez les moutons, alors qu'il n'y a pas eu de différence notable chez les génisses.

\section{Temps de latence}

Le temps de latence, séparant la fin des grands repas du début de la rumination, a été toujours plus élevé pour les moutons que pour les génisses. L'augmentation de la longueur des brins d'ensilage a entraîné un allongement de cette durée pour les deux espèces animales. La suppression du conservateur a fait diminuer le temps de latence chez les moutons, mais l'a augmenté chez les génisses.

\section{Durée journalière de rumination}

Les génisses ont passé plus de temps à ruminer que les moutons les ensilages avec conservateur, mais le même temps, les fourrages verts et les ensilages sans conservateur. L'augmentation de la longueur des brins s'est traduite par une baissc significative de la durée de rumination chez les deux espèces. La suppression du conservateur n'a pas eu d'effet chez les moutons et a entraîné une baisse de cette durée chez les génisses. 


\section{Durée unitaire de rumination}

Les durées unitaires de rumination ont toujours été plus faibles pour les génisses. Le remplacement des ensilages à brins courts par ceux à brins longs a entraîné une forte augmentation de ces durées unitaires chez les moutons, mais pas chez les génisses. La suppression du conservateur a eu un léger effet positif chez les deux espèces, effet cependant non significatif pour les moutons.

Les cycles de rumination ont été plus courts pour les ensilages à brins longs que pour ceux à brins courts. Nous l'avons vérifié pour les ensilages correspondant au fourrage $\mathrm{n}^{\circ} 2: 43$ secondes chez le mouton pour l'ensilage à brins longs contre 47 secondes pour celui à brins courts, 48 et 52 secondes chez les génisses. En outre, chez les animaux recevant des ensilages à brins longs, nous avons observé de nombreux cycles de rumination sans remontée de bols alimentaires. Ces cycles se caractérisent surtout par un nombre très réduit de coups de mâchoire. Cette " pseudo-rumination " a été très importante chez les moutons, mais moindre chez les génisses. En moyenne, 38 p. 100 des cycles de rumination ont été anormaux chez les moutons contre seulement 11 p. 100 chez les génisses. La «pseudo-rumination » s'est toujours située au cours des premières heures suivant la fin des grands repas. Ainsi, après déduction du temps de "pseudo-rumination " la durée unitaire de rumination des ensilages à brins longs a été relativement proche de celle des ensilages à brins courts : soit 9,12 et $10,76 \mathrm{~mm} / \mathrm{g} / \mathrm{kg} \mathrm{P}^{0,7,}$ pour les moutons et 6,60 et 6,62 pour les génisses (fourrages $1,2,3$ et 8 ).

\section{B. Relations entre la qualité de conservation des ensilages et le comportement des animaux}

La longueur exacte des brins d'ensilage n'a pas été mesurée. Nous n'avons donc pu étudier les relations entre leur longueur et le comportement des animaux. Par contre, pour tous les ensilages nous disposons d'une analyse précise de la qualité de conservation. Nous avons donc relié le comportement des animaux recevant les ensilages à brins courts (à cause de leur nombre suffisant; 12 sont pris en compte) à celui des mêmes animaux recevant les fourrages verts correspondants et à la qualité de conservation des ensilages.

Chez le mouton, les paramètres suivants pour l'ensilage sont liés étroitement aux mêmes paramètres pour le fourrage vert : la quantité ingérée par jour (cf. plus haut), la durée journalière d'ingestion, la durée unitaire d'ingestion, la quantité ingérée par grands repas, la durée d'ingestion lors de ces grands repas et la durée unitaire d'ingestion pendant ces grands repas, mais ni la teneur en acide acétique, ni celle en $\mathrm{N}-\mathrm{NH}_{3}$ (p. $100 \mathrm{~N}$ total) n'ont d'effet significatif.

Chez la génisse, sur ces 6 paramètres, seuls 2 (durée journalièrce d'ingestion et durée unitaire d'ingestion) sont reliés significativement au même paramètre pour le fourrage vert; par contre l'effet de l'acide acétique est significatif sur tous sauf sur la durée d'ingestion des grands repas. Les effets de l'acide acétique sont négatifs sur la quantité ingérée par jour et par grands repas et positifs sur la durée journalière d'ingestion et les durées unitaires d'ingestion par jour et par grands repas. En outre la teneur en $\mathrm{N}-\mathrm{NH}_{3}$ a un effet positif significatif sur la durée journalière d'ingestion. 
C. Liaisons entre les paramètres du comportement des moutons et ceux des génisses (tabl. 8)

Nous avons étudié les liaisons entre les paramètres du comportement du mouton et ceux de la génisse pour le groupe homogène le plus important d'ensilages, c'est-à-dire à brins courts additionnés d'acide formique. Ces liaisons sont relativement étroites pour les quantités ingérées (fig. 3), les durées d'ingestion par grands repas, ainsi que pour les durées unitaires d'ingestion et de rumination.

\section{Tableau 8}

Liaisons entre les paramètres du comportement des moutons ( $x$ ) et des génisses (y). (10 ensilages à brins courts + acide formique).

Relationships between parameters of feeding behaviour in sheep $(x)$ and heifers $(y)$. (10 chopped silages + formic acid).

\begin{tabular}{|c|c|c|}
\hline & $\begin{array}{l}\text { Coefficient } \\
\text { de corrélation } \\
\text { Correlation } \\
\text { coefficient }\end{array}$ & $\begin{array}{l}\text { Ecart-type résiduel } \\
\text { Standard deviation }\end{array}$ \\
\hline $\begin{array}{l}\text { Quantité ingérée/jour } \ldots \ldots \ldots \\
\text { Voluntary intake/day }\end{array}$ & $0,791^{*}$ & 4,0 \\
\hline $\begin{array}{l}\text { Durée journalière d'ingestion } \ldots \ldots \ldots \ldots \ldots \\
\text { Daily time spent eating }\end{array}$ & 0,450 & 52 \\
\hline $\begin{array}{l}\text { Durée unitaire d'ingestion } \ldots . \\
\text { Unitary eating time }\end{array}$ & $0,734 *$ & 0,58 \\
\hline $\begin{array}{l}\text { Durée journalière de rumination } \ldots \\
\text { Daily time spent ruminating }\end{array}$ & 0,349 & 49 \\
\hline $\begin{array}{l}\text { Durée unitaire de rumination } \ldots \ldots \ldots \ldots \cdots \\
\text { Unitary ruminating time }\end{array}$ & $0,693 *$ & 0,56 \\
\hline $\begin{array}{l}\text { Nombre total de repas/jour } \ldots \ldots \ldots \ldots \\
\text { Total number of meals/day }\end{array}$ & 0,527 & 1,2 \\
\hline $\begin{array}{l}\text { Par grands repas: } \\
\text { Per main meals }\end{array}$ & & \\
\hline $\begin{array}{l}\text { - Quantité ingérée } \ldots \ldots \ldots \ldots \ldots \ldots \ldots \\
\text { Feed intake }\end{array}$ & 0,290 & 5,2 \\
\hline $\begin{array}{l}\text { - Durée d'ingestion } \ldots \ldots \ldots \ldots \ldots \ldots \ldots \\
\text { Time spent eating }\end{array}$ & $0,680^{*}$ & 14 \\
\hline $\begin{array}{l}\text { - Durée unitaire d'ingestion } \ldots . . . . \\
\text { Unitary eating time }\end{array}$ & 0,060 & 0,45 \\
\hline $\begin{array}{l}\text { * Valeurs significatives }(\mathrm{P}<0,05) \\
\text { Significant values }(P<0.05)\end{array}$ & & \\
\hline
\end{tabular}




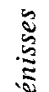

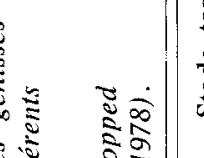

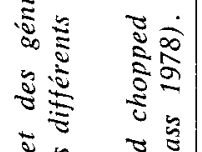

3

0

25

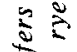

吾

s.

$2 \stackrel{2}{2}$

范

茫

?

है

ป

- $5 \frac{2}{20} \div$

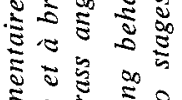

공ำ

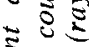

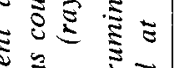

ริำ

文公

¿ 0

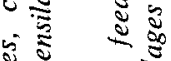

ए 0

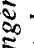

$\stackrel{\Xi}{\Xi}$

范

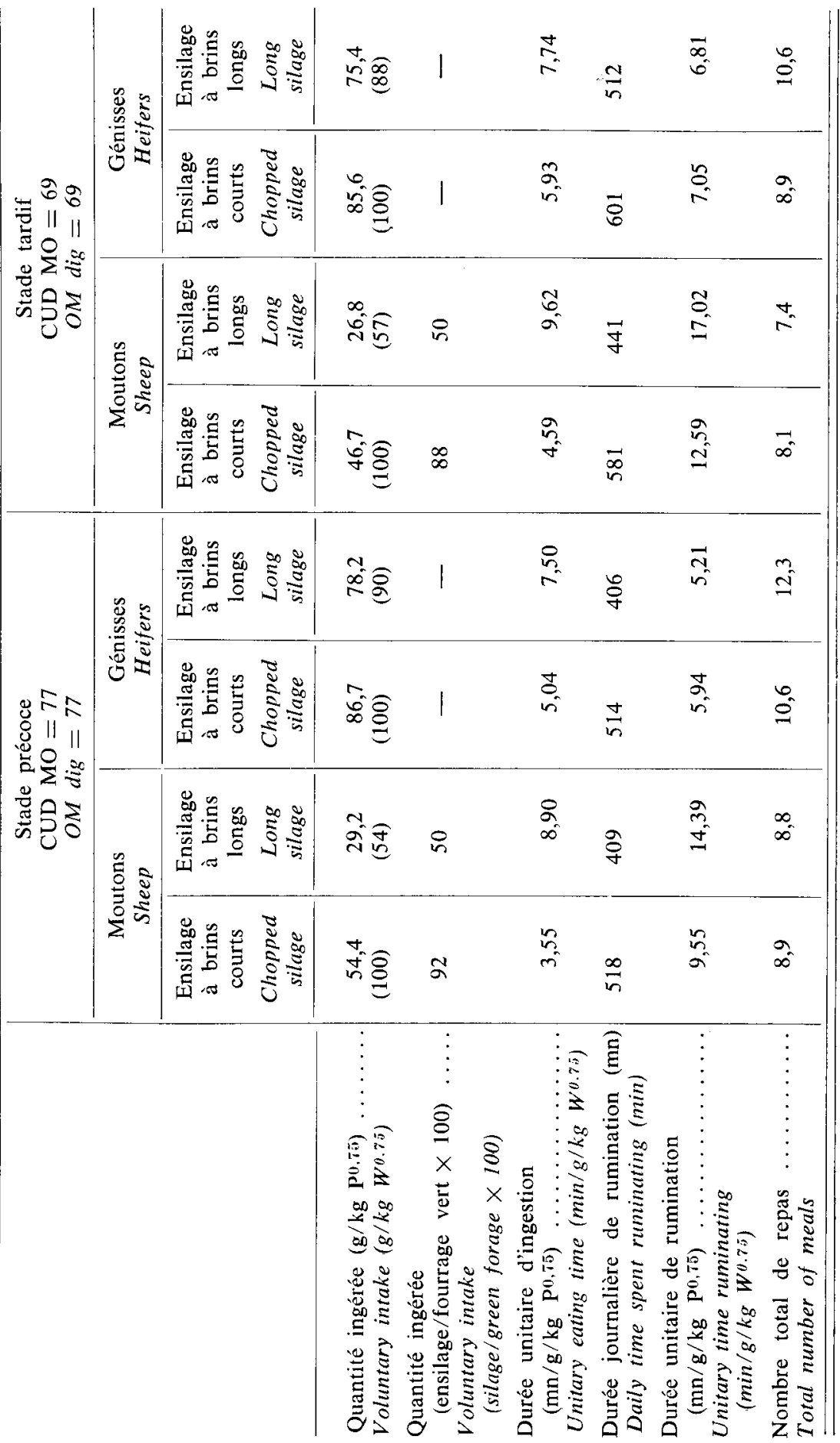




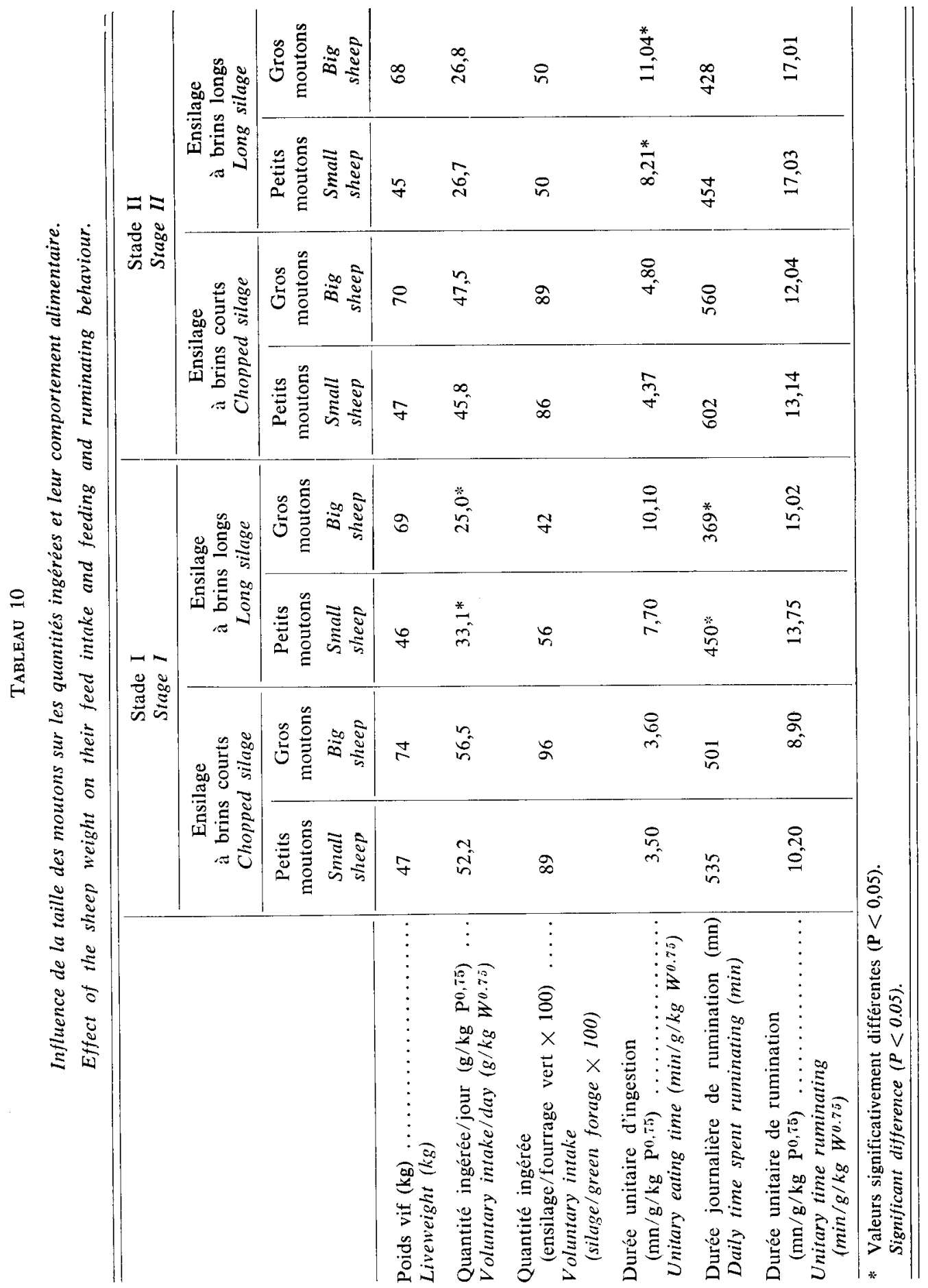


D. Influence du stade de récolte sur l'effet de la finesse de hachage

(tabl. 9)

Les quantités de MS ingérées ont légèrement baissé pour les moutons avec le stade de récolte, mais pour les deux types d'animaux, le rapport :

quantité ingérée brins longs

quantité ingérée brins courts

n'a pas été modifié. Par contre, les durées unitaires d'ingestion et de rumination ainsi que les durées de rumination, ont augmenté, lorsque la digestibilité a baissé. Le nombre de repas $a$, par contre, légèrement baissé.

\section{E. Influence de la taille des moutons (tabl. 10)}

Il n'y a pas eu d'influence significative de la taille des moutons sur les quantités ingérées (en $\mathrm{g}$ de $\mathrm{MS} / \mathrm{kg} \mathrm{P}^{\mathbf{0}, 75}$ ) d'ensilage à brins courts, ni sur les paramètres correspondants du comportement alimentaire.

\section{Discussion}

Les résultats obtenus confirment l'influence de la finesse de hachage sur les quantités d'ensilage ingérées par les ovins (Dulphy \& Demarouilly, 1973; DeswySEN, Vanbelle \& Focant, 1978) et par les bovins (Lindell et al., 1970) ; Dulphy \& Demarouilly, $1975 \mathrm{a}$ et $\mathrm{b}$ ). Ils indiquent :

- la moins grande sensibilité des bovins par rapport aux ovins à la finesse de hachage des ensilages (Demarouilly \& Dulphy, 1977);

- la plus grande sensibilité des bovins à la qualité de conservation ;

- l'ingestion par les moutons des ensilages à brins courts, même très bien conservés, en quantité plus faible que celle du fourrage vert de départ.

La finesse de hachage des ensilages d'herbe modifie leur ingestibilité (MrchaleTDoREAU, 1975 ; DESWYSEN, 1980) en intervenant au moment de l'ingestion, puis au cours de la rumination.

Dans le cas des ensilages à brins longs la durée unitaire d'ingestion augmente. Le mouton s'efforce de réduire, dès l'ingestion, la longueur des brins d'ensilage ainsi que le montre l'étude de la granulométrie des bols alimentaires (MichaletDOREAU, 1975). La taille moyenne des particules dans les bols est pratiquement identique quelle que soit la longueur des brins de l'ensilage offert. Les différences de quantités ingérées entre ensilages à brins courts et longs sont donc probablement liées au fait que le mouton n'ingère les ensilages à brins longs qu'après les avoir 
réduits en brins relativement courts. Une mastication plus poussée réduit d'autant la vitesse d'ingestion des ensilages. Chez la génisse, la diminution de la vitesse d'ingestion est moins importante en raison soit d'une mastication plus efficace que celle des moutons, soit d'une moindre réduction de la taille des brins.

La rumination chez le mouton recevant un ensilage à brins longs est perturbée (Michalet-Doreau, 1975 ; Deswysen, 1980). En effet, malgré une mastication plus poussée, quelques longues particules arrivent dans le rumen, gènent les mouvements des autres particules (DESWYSEN, 1980) et rendent plus difficile la régurgitation des bols méryciques (DESWYSEN \& EHRLEIN, 1981). Les perturbations qui en résultent (augmentation du temps de latence et pseudo-rumination) sont suffisantes pour entraîner une baisse supplémentaire des quantités d'ensilage ingérées (DulPHY \& DEmarouilly, 1973). Dans les essais de Michalet-Doreau (1975) l'examen des contenus de rumen de moutons recevant des ensilages à brins longs n'a cependant pas révélé la présence de longues particules : les perturbations du comportement mérycique pourraient résulter alors simplement de la faible quantité ingérée d'ensilage. Le mouton ne compense pas, en effet, sa faible vitesse d'ingestion par une durée journalière d'ingestion accrue et le rumen ne serait donc pas assez rempli. Au demeurant la rumination n'est que fort peu perturbée chez la génisse en raison, probablement, de différences anatomiques au niveau du rumen, favorisant la circulation des particules, et de la plus grande taille de l'œsophage rendant l'animal moins sensible à la présence éventuelle de longues particules dans les bols alimentaires.

La qualité de conservation des ensilages modifie les quantités ingérées (Mc DoNALD, 1981). Il existe en effet des liaisons négatives significatives entre les quantités d'ensilage ingérées et les teneurs en acide acétique (WILkıns et al., 1971 ; DemarQUILLY, 1973). Cependant un apport d'acide acétique lors de la distribution ne modifie guère les quantités ingérées d'un ensilage donné (Hutchinson \& WiLkins, 1971; DESWYSEN, 1980).

Les génisses, plus sensibles que les moutons à la qualité de conservation des ensilages, réduisent leur vitesse d'ingestion et les quantités ingérées par grands repas. L'origine de cette réaction est peut-être d'origine gustative et/ou olfactive (GoATcher \& $\mathrm{CHuRCH}, 1970$; Church, 1971).

Un fait essentiel est la différence de réaction entre ovins et bovins recevant d'excellents ensilages (à brins courts et très bien conservés) par comparaison aux fourrages verts correspondants. Les moutons ingèrent 15 à 20 p. 100 de matière sèche en moins sous forme d'un excellent ensilage que sous forme de fourrage vert (Demarouilly, 1973 ; Dulphy \& Demaroullly, 1973 ; Dulphy \& Michalet-Doreau, 1981). Ce phénomène est lié à une diminution de la durée des grands repas. Le rassasiement rapide dans le cas d'ensilages ne semble pas être lié à des problèmes gustatifs ou olfactifs : les ensilages biens conservés sont ingérés en effet plus rapidement que les fourrages verts. Il est peut-être d'ordre métabolique par l'intermédiaire de chémorécepteurs ruminaux ou post-ruminaux. Le mouton pourrait présenter une sensibilité à l'acide lactique (Bueno, 1975 ; Morgan \& L'Estrange, 1976) ou à l'ensemble des acides organiques (DESWYSEN, 1980) plus grande que la génisse, bien que ceci soit contradictoire avec le fait que le mouton est moins sensible à la qualité de conservation que la génisse.

Nous avons, dans nos essais, mesuré l'effet de deux facteurs annexes : le stade de récolte de l'ensilage et le poids des moutons. Pour le premier facteur notre hypo- 
thèse était que l'avancement du stade de récolte, en augmentant la teneur en constituants pariétaux de la plante, pouvait accentuer l'effet de la longueur des brins. Le stade de récolte, en accord avec ANDERson (1982), n'a cependant pas modifié l'effet de la longueur des brins, mais l'avancement de ce stade tend à faire baisser les quantités ingérées par les moutons. Pour le second facteur, notre hypothèse était que l'augmentation de la taille de l'animal expérimental pouvait diminuer l'effet de la longueur des brins, mais la taille des moutons n'a, apparemment, pas eu d'effets majeurs.

Les méthodes de travail utilisées font appel à la mesure des quantités de fourrage vert ingérées à deux époques très différentes de l'année. Or les niveaux d'ingestion seraient plus élevés en jours longs qu'en jours courts pour des béliers castrés ou non, cela d'après les observations de plusieurs auteurs (GoRdon, 1964 ; TARTTELIN, 1968 ; Milne et al., 1976 et 1978 ; KAY, 1979 ; Brown et al., 1979). Dans nos essais, une partie des différences de réaction entre moutons et génisses est peut-être liée à une moindre réaction de la seconde espèce envers les facteurs saisonniers.

En définitive, les facteurs (finesse de hachage, qualité de conservation) qui modifient les quantités d'ensilage ingérées ne peuvent être extrapolés d'une espèce animale à l'autre. Cela implique que des essais effectués pour étudier puis prévoir les quantités ingérées par des bovins doivent l'être chaque fois sur l'espèce concernée tant que les liaisons entre ovins et bovins par catégorie d'ensilage ne seront pas mieux connues. Nous avons seulement pu établir de telles liaisons pour des ensilages à brins courts additionnés d'acide formique (Dulphy \& Michalet-Doreau, 1981).

\section{Summary}

\section{Comparative study of feed intake, feeding and ruminating behaviour in sheep and cattle fed silages subjected to different treatments}

Twenty silages (tabl. 1 and 2) were given to sheep and heifers : 10 silages harvested with a precision-chop machine and treated with formic acid, 6 silages harvested with a flail-machine and treated with formic acid, 4 silages harvested with a precision-chop machine without preservative. These silages were made from 10 fresh forages, 8 of them had been fed to comparable animals during the harvest.

Comparisons concerning feed intake, feeding and ruminating behaviour were made for a better understanding of the variations in feed intake with animal species and method of silage.

All the forages were ingested more by heifers than by sheep and the best silages (short particles, formic acid) were consumed at the same level than fresh forages by the former, but in smaller quantity ( 86 p. 100) by the latter (fig. 1 and 2).

Heifers were less affected by the length of silage-particles, which decreased silage intake, than sheep ( -16 p. 100 versus -41 p. 100), but more by the absence of formic acid, which decreased preservation quality and thus silage intake (Tabl. 3, 4 and 5) (- 19 p. 100 vs -13 p. 100$)$.

Heifers ingested during a longer time than sheep, but the parameters leading to the best understanding of the intake variations between the two species and their modifications with the silage method wcre the unitary eating time $\left(\mathrm{mn} / \mathrm{g} / \mathrm{kg} \mathrm{W}^{0.75}\right)$ and the quantity of dry matter consumed during the two main meals immediately after silage feeding.

Sheep ingested the silages with short particles and well preserved as rapidly as fresh forages, but were quickly satieted during the main meals and were not able to compensate 
during the small meals (tabl. 6). For these silages this phenomenon of satiety did not appear in heifers.

When the length of silage particles increased the unitary eating time strongly increased in sheep and rumination was disturbed. These phenomena were reduced in heifers.

The decrease in silage preservation quality increased the unitary eating time and clearly decreased the feed intake during the main meals in heifers. These phenomena were also observed in sheep, but to a less extent.

Other parameters of feeding and ruminating behaviours were examined (tabl. 6) : number of meals per day, characteristics of the small meals, latency time between the end of the main meals and the beginning of rumination, time spent ruminating.

The time spent searching for feed was shorter in heifers than in sheep and in the latter the number of jaw-movements per $\mathrm{g}$ of ingested forage was low (tabl. 7).

In sheep the behaviour parameters of the animals fed silages were closely related to those of animals fed fresh forages. This was not true in heifers which reacted more to preservation quality.

When the animals were fed the short, well preserved, silages only some parameters were closely related between sheep and heifers (tabl. 8) : daily dry matter intake (fig. 3), unitary eating and ruminating times and duration of the main meals.

The growth stage of the ensiled forage did not affect our observations and conclusions (tabl. 9), nor did the weight of the sheep (tabl. 10).

The results are discussed in connection with the problem of regulation of silage intake by ruminants.

Key words : Grass silage, sheep, cattle, intake, feeding behaviour.

Reçu en mars 1982.

Accepté en mars 1984.

\section{Références bibliographiques}

ANDERSON R., 1982. Effect of stage of maturity and chop length on the chemical composition and utilization of formic acid-treated rye-grass and formic acid silage by sheep. Grass and Forage Science, 37, 139-145.

Brown W.B., Forbes J.M., Goodall E.D., Kay R.N.B., Simpson A.M., 1979. Effect of photoperiod on food intake, sexual condition and hormone concentration in stags and rams. J. Physiol., 296, 58-59.

Bueno L., 1975. Rôle de l'acide DL lactique dans le contrôle de l'ingestion alimentaire chez le mouton. Ann. Rech. Vét., 6, 325-336.

Church D.C., 1971. Taste, appetite and regulation of food intake. In : Digestive physiology and nutrition of ruminants. Vol. 2, Nutrition, 737-762.

De Brabander D.L., Aerts J.V., Boucque C.V., Buysse F.X., 1976. Influence de la longueur du hachage de l'herbe ensilée sur l'ingestion chez les vaches laitières. Revue de l'A griculture, 29, 341-354.

Demarquilly C., 1973. Composition chimique, caractéristiques fermentaires, digestibilité et quantité ingérée des ensilages de fourrages : modifications par rapport au fourrage initial. Ann. Zootech., 22, 1-35.

Demarquilly C., Dulphy J.P., 1977. Effect of ensiling on feed intake and animal performance. Proc. of the Int. Meeting on Animal Production from temperate Grassland, Dublin, June, 53-61. 
DESWYSEN A., 1980. Influence de la longueur des brins et de la concentration en acides organiques des ensilages sur l'ingestion volontaire chez les ovins et bovins. Thèse. Université Catholique de Louvain, $254 \mathrm{p}$.

Deswysen A., EhrLein H.J., 1981. Silage intake, rumination and pseudo-rumination activity in sheep studied by radiography and jaw movement recordings. Br. J. Nutr., 46, 327-335.

Deswysen A., Vanbelle M., Focant M., 1978. The effect of silage chop length on the voluntary intake and rumination behaviour of sheep. J. Br. Grassld. Soc., 33, 107-115.

Duckworth J.E., Shirlaw D.W., 1958. A study of factors affecting feed intake and the eating behaviour of cattle. Ann. Behav., 6, 147-154.

Dulphy J.P., Demarquiley C., 1973. Influence de la machine de récolte et de la finesse de hachage sur la valeur alimentaire des ensilages. Ann. Zootech., 22, 199-217.

Dulphy J.P., Demarquilly C., 1974. Etude du comportement alimentaire et mérycique de moutons recevant des fourrages verts hachés. Ann. Zootech., 23, 193-212.

Dulphy J.P., Demarquilly C., 1975 a. Influence de la machine de récolte sur la valeur des ensilages de graminées pour les génisses de race laitière. Ann. Zootech., 24, 351-362.

Dulphy J.P., Demarquilly C., 1975 b. Influence de la machine de récolte sur les quantités d'ensilage ingérées et les performances des vaches laitières. Ann. Zootech., 24, 363-371.

Dulphy J.P., Demarquilly C., 1977. Influence de l'addition d'acide formique sur la valeur des ensilages de graminées pour les génisses. Ann. Zootech., 26, 45-57.

Dulphy J.P., Demarquilly C., Henry Martine, 1975. Perte de composés volatils lors de la détermination à l'étuve de la teneur en matière sèche des ensilages. Ann. Zootech., 24, 743-756.

Dulphy J.P., Lienard G., Andrieu J.P., Garel J.P., 1983. Intérêt d'une nouvelle ensileuse à double-coupe : étude dans une zone de demi-montagne et conséquences économiques pour la production de lait. Bull. Tech. C.R.Z.V. Theix, I.N.R.A., 51, 31-42.

Dulphy J.P., Michalet-Doreau b̈rigitte, 1975. Influence comparée de la machine de récolte sur les quantités d'ensilage ingérées par des génisses et des moutons. Ann. Zootech., 24, 757-763.

Dulphy J.P., Michalet-Doreau Brigitte, 1981. Prévision de l'ingestibilité des ensilages d'herbe. In : Prévision de la valeur nutritive des aliments des ruminants. Ed. I.N.R.A. Publications, route de Saint-Cyr, 78000 Versailles, 169-188.

Dulphy J.P., Michalet-Doreau Brigitte, 1983. Comportement alimentaire et mérycique d'ovins et de bovins recevant des fourrages verts. Ann. Zootech., 32, 465-474.

Gill J., Campling R.C., Westgarth D.R., 1966. A study of chewing during eating in the cow. Br. J. Nutr., 20, 13-23.

Goatcher W.D., Church D.C., 1970. Review of some nutritional aspects of the sense of taste. J. Anim. Sci., 31, 973-981.

Gordon J.G., 1964. Effect of time of the year on the roughage intake of housed shecp. Nature, 204, 798-799.

Hutchinson K.J., Wilkins R.J., 1971. The voluntary intake of silage by sheep. 2. - The effects of acetate on silage intake. J. Agric. Sci., Camb., 77, 539-543.

KAY R.N.B., 1979. Seasonal changes of appetite in deer and sheep. A.R.C. Res. Rev., 5, $13-15$.

Lindell L., LingVall P., Schmekel J., Wiktorsson H., 1970. The chopped length of silage. The influence on silage quality, and on the feed consumption, the production and health of cows. Jordbrukstekniska Institutet. Meddelande $\mathrm{nr} 334$.

Mc Donald P., 1981. The biochemistry of silage, 226 p., John Wiley and Sons Ed.

MichaleT-DoREAU Brigitte, 1975. Recherches sur les causes de variation des quantités d'ensilage d'herbe ingérées par les ruminants. Thèse de Docteur-Ingénieur. Université de Nancy.

Milne J.A., McRae J.C., Spence A.M., Wilson S., 1976. Intake and digestion of hill land vegetation by the red deer and the sheep. Nature, 263, 763.

Milne J.A., Mc Rae J.C., Spence A.M., Wilson S., 1978. A comparison of the voluntary intake and digestion of a range of forage at different times of the year by the sheep and the red deer. Br. J. Nutr., 40, 347-357. 
Morgan D.J., L'Estrange J.L., 1976. Effect of dietary additions of hydrochloric and lactic acid on feed intake and metabolism of sheep and cattle. Ir. J. Agric. Res., $15,55-63$.

Murdoch J.C., 1965. The effect of length of silage on its voluntary intake by cattle. J. Br. Grassld. Soc., 20, 54-58.

RUCKEBUSCH Y., 1963. Recherches sur la régulation centrale du comportement alimentaire chez les ruminants. Thèse Doct. Sci., Université de Lyon.

Saue O., Breirem K., 1969. Formic acid as a silage additive. Proc. 3rd General Meeting European. Grassland Federation. Braunschweig, 161-172.

TartTElin M.F., 1968. Cyclical variations in food and water intakes in ewes. J. Physiol., 195, 29-31.

Thomas P.C., Kelly N.C., Wait M.K., 1976. The effect of physical form of a silage on its voluntary consumption and digestibility of sheep. J. Br. Grassld. Soc., 31, 19-22.

Waldo D.R., 1973. Chemical preservation of forages. Proc. Cornell. Nutr. Conf. for feed Manufact., $50-58$.

Wilkins R.J., Hutchinson K.J., Wilson R.F., Harris C.E., 1971. The voluntary intake of silage by sheep. I. - Interrelationships between silage composition and intake. J. Agric. Sci., Camb., 77, 531-537.

WiLSON R.F., WiLKins R.J., 1973. Formic acid as a silage additive for wet crops of cocksfoot and lucerne. J. Agric. Sc. Camb., 80, 225-231.

WILSON R.K., FLYNN A.V., 1974. Observation on the eating behaviour of individually fed beef cattle offered grass silage ad libitum. Ir. J. Agric. Res., 13, 347-349. 